\title{
Simplified VIV Response of Partially Strake-Covered Pipe Spans
}

\author{
by Ralf Peek ${ }^{1}$, Jie Wu², Decao Yin ${ }^{2}$, Malcolm Carr ${ }^{3}$, Sze-Yu Ang ${ }^{4}$ and Chiara Bernardo ${ }^{5}$
}

\begin{abstract}
A simplified method for the fatigue assessment for partially strake-covered pipeline spans is provided and calibrated so that for the bare pipe case it is consistent with the current DNVGL Recommended Practice (DNVGL-RP-F105). It is based on a Rayleigh-Ritz approximation using the first undamped mode shape. In that sense it is equivalent to a single-mode analysis using Shear7, except that it accounts for frequencydependent added mass, and the formulation is presented in a compact and convenient non-dimensional form. The method presented may also be considered a simplification of that in Vivana in that it does not account for propagating wave effects, and is therefore named "Sivana".
\end{abstract}

Like any frequency-domain method Sivana is based on the assumption of harmonic response. This requires excitation and added mass functions that define a harmonic hydrodynamic force as a function of the harmonic motion. On the other hand, current practice by DNVGL-RP-F105 is based on a response function, which gives VIV amplitude as function of reduced velocity and the Scruton mass-damping parameter $\mathrm{K}_{\mathrm{s}}$. To bridge this gap, an inversion technique is developed whereby the excitation function for bare pipe is derived from the DNVGLRP-F105 response function, such that for bare pipe the Sivana calculation will lead to the same response amplitudes as DNVGL-RP-F105.

The Sivana method is illustrated by an example involving a single span with different degrees of strake coverage centered at midspan.

Although the Sivana method might be considered a natural and straight-forward extension of current span assessment practice to include partial strake coverage, caution is in order for certain approximations in the DNVGL-RP-F105 response function may be less appropriate for the use in this paper, than for the use envisioned by the developers of the DNVGL guidance.

\section{Introduction}

Pipeline spans can arise from scour, as well as from laying the pipe on an uneven seabed. The former can involve high overall costs to monitor span formation and remediate when necessary, to prevent a fatigue failure due to VIV.

For scour-induced spans that become too long, providing a support near midspan may temporarily alleviate the problem by dividing the span in two shorter ones, but further scour can quickly lead to two spans of excessive length. The supports also disrupt a natural cycle whereby spans finally become so long that the pipe sags into the trench it creates. A better alternative can be to cover the span with strakes, partially, to the extent needed to limit VIV fatigue damage. The method presented in this paper can not only account for partial strake coverage, but also for other changes along the length of the span, such as changes in current velocity and/or pipe diameter.

The response function approach is described in detail in DNVGL-RP-F105 [1]. By fitting VIV test data on flexible pipes or spring-supported rigid cylinders, the response function provides the VIV amplitude as a function of the component of current velocity normal to the pipeline, the still-water undamped natural frequency, the mass damping ratio, and the hydraulic diameter of the pipe.

In addition to the above primary parameters, secondary parameters that affect the VIV response amplitude for each mode calculated according to F105 [1] include: For cross-flow: certain effects of seabed proximity and scour trenches on VIV and the frequency ratio for modes that may "compete". For in-line: the turbulence of the incoming flow, and the angle between the flow direction and the pipeline. In addition, for both in-line and

\footnotetext{
${ }^{1}$ Peek Solutions, Sant Andreu de Llavaneres, Spain.

${ }^{2}$ Sintef Ocean, Trondheim, Norway

${ }^{3}$ Crondall Energy Subsea, Newcastle, UK.

${ }^{4}$ Shell Global Solutions International, B.V., Rijswijk, The Netherlands.

${ }^{5}$ Shell Philippines Exploration, B.V., Manila, Philippines.
} 
cross-flow, there are the unsteady flow parameters " $\alpha$ " and "KC" in F105 arising from including wave as well as steady current effects.

Finally there are parameters that are "not explicit"6 in F105, in the sense that they are considered in developing the response function but the sensitivity to variations in that parameter in the range of application has apparently has been neglected, presumably deemed to be adequately covered by safety margins included explicitly (in the form of safety factors) or implicitly (e.g. in the form of a response function that provides an envelope to the available data). These include the Reynolds number, pipe roughness, the pipe mass (except to the extent that the pipe mass influences the still-water natural frequency), the mode shape and "in-line induced cross-flow", which refers to how the presence of an in-line line mode can influence cross-flow response.

A drawback of the response function approach is that changes in current velocity, pipe diameter, and strake coverage along the length of the pipeline cannot be accounted for, except if the response function is constructed from tests involving the same changes along the length of the pipeline.

The alternative is to rely more on theory and less on tests, as is done for risers, using codes such as Shear7 [2], VIVA [3], or Vivana [4,5]. For this, the empirical basis comes from forced vibration tests, which represent a single cross section. The motion is assumed to be harmonic and either horizontal (in-line) or vertical (crossflow). This is captured in unidirectional, harmonic forced-vibration tests on a rigid cylinder such as those of [6] for cross-flow, and [7] for in-line. The excitation and added mass functions resulting from such tests are then used in the aforementioned codes to calculate VIV response. This is referred to as the excitation function approach. Sivana [8] is a simplified version of this excitation function approach, based on a Rayleigh-Ritz approximation using the undamped, still-water modes. For pipeline spans responding in the lowest-frequency modes with negligible propagating wave effects this provides a good approximation. In Section 3, the energy balance condition for Sivana is given in a convenient non-dimensional form.

For pure in-line VIV, the excitation function approach has been quite successful: using the excitation and added mass functions from tests by Aronsen [7], a good approximation to the in-line response function from F105 [1] is obtained with Sivana for the conditions considered in [8] (including a mass ratio of 1.23, low turbulence, normal flow and no seabed proximity effects), as shown in Figure 1.

However, it gets more complicated at current velocities beyond the onset of cross-flow VIV, since cross-flow ("CF") and in-line ("IL") VIV interact: Due to the hydrodynamic interaction", the CF motion typically includes a component of IL motion at double the CF frequency [22, 23]. Further, the IL component can enhance the CF excitation leading to higher CF response than would occur otherwise. To account for this and other effects, cross-flow excitation functions in Vivana are not obtained directly from forced vibration tests such as [6]. They have been adjusted to account for these effects [24,5]. Despite this adjustment, carried out mainly with riser response in mind, the excitation and added mass functions from Vivana [4] do not provide a good match to the DNVGL-RP-F105 cross-flow response function is seen in Figure 2.

To investigate CF-IL interactions, tests involving forced simultaneous forced motions in both directions have been performed [7]. However, even if one assumes that the in-line frequency is double the cross-flow frequency, and neglects Reynolds number effects, it still takes 4 dimensionless parameters to define such a bidirectional test: an IL amplitude and a phase difference are added to the CF amplitude and frequency. With 4 instead of 2 parameters, it requires orders of magnitude more tests to empirically define the excitation and added mass functions in 4 variables, since enough experimental points in the 4-dimensional space are needed so that one can reasonably interpolate between those points. For these reasons, bi-directional forced vibration tests may yield interesting and useful insights, but they are not the most direct path to a practical yet reasonably accurate assessment procedure, nor are they intended as such.

Another approach to obtain excitation and added mass functions is based on the response of a densely

6 Only the Reynolds number is stated to be "not explict" in F105 [1], but the other parameters mentioned also do not influence the F105 calculated response, and it is assumed that they been considered in a similar way.

7 In addition to hydrodynamic interaction, there can be structural interaction between IL and CF directions, to the extent that the drag force makes the pipe hang in in inclined plane, rather than a vertical plane as it would under gravity force alone. The modes are then in-plane and normal to the plane in which the pipe hangs. Further interactions can occur due to geometrically nonlinear dynamic structural behaviour. However, here reference is made only to the hydrodynamic interaction, which is also present when the dynamic behavior remains purely in the linear domain with non IL-CF interactions. 
instrumented flexible pipe, by deducing the hydrodynamic forces from the stiffness and motion of the pipe. Thus, $\mathrm{Wu}[9]$ estimated hydrodynamic coefficients along the pipe from a limited number of acceleration and strain measurements using a finite element beam model for a pinned-pinned beam under constant tension. The forces on the pipe are adjusted to optimize the fit to the experimental results, with the dynamic equilibrium equation imposed as a constraint. From the resulting hydrodynamic forces, local excitation and added mass coefficients are estimated. However, the application of the method to the free spanning pipe needs to be further investigated, especially for low reduced velocities, and the associated increased uncertainty due to small measured response amplitudes.

For the above reasons, the approach used here to estimate the excitation function is a different one: It builds on established engineering practice that has already been extensively calibrated against experimental results. Instead of obtaining the excitation function from forced vibration tests, it is obtained by inversion of the response function in DNVGL-RP-F105 [1], which is based on VIV tests on flexible and/or spring supported pipes, and for cross-flow already accounts for the bi-directional, non-harmonic components of VIV motion. The direct inversion is enabled by the Sivana simplifications and presented for cross-flow in Section 5. It leads to an excitation function that exactly reproduces the response predicted by DNVGL-RP-F105 [1] for uniform pipe and current velocity. Combining this effective excitation function for bare pipe with existing results for straked parts, then yields the cross-flow VIV response for the partially straked pipe.

Seabed proximity effects can be important [10-15], but are not considered here. All test results used, including forced vibration tests, and tests on flexible pipes, are for pipes or cylinders "towed" in stationary water at a sufficient distance to boundaries to make the boundary effects negligible. Similar principles can be applied with seabed proximity effects, but that would require a repeat of test programs such as [6,7] in flowing water at different distances from the model seabed, possibly also considering different roughness of the seabed. Here only the effect of partial strake coverage on VIV is addressed without considering seabed proximity effects at the same time.

\section{Nomenclature}

\subsection{Terms and Abbreviations}

2D test refers to a VIV test on a spring-supported rigid cylinder that is free to move with constant displacement along its length.

bare "bare pipe" refers to the pipe that may be coated but is not covered with strakes

CF cross-flow, refers to component of VIV motion normal to the flow direction and the pipe

F105 refers to the DNVGL Recommended Practice for span assessment [1]

F105 added mass function: refers to added mass function given in Section 4.4.15 of [1]; with the added mass coefficient under flowing conditions, $C_{a}$, given as a function of reduced velocity $V_{R}=1 / f_{s}^{*}$.

F105 response function: response function $A^{*}=A^{*}\left(V_{R}, K_{s}\right)$ recommended in Section 4.6 of [1] for in-line VIV, and in Section 4.4 of [1] for cross-flow VIV. Specifically it refers to this function for steady currents, with all safety factors set to 1 , no seabed proximity effects, low turbulence, flow normal to the pipeline and no mode competition. There are no other secondary parameters that influence the calculated response in [1], as described in the introduction.

IL in-line, refers to component of VIV motion in the flow direction (where the flow is not normal to the pipe it refers to the component of VIV motion normal to the pipe and the CF direction of motion)

normalized velocity: current velocity expressed in pipe diameters per period of VIV oscillation under flowing conditions, $1 / \mathrm{f}^{*}$

reduced velocity: current velocity expressed in pipe diameters per natural period of oscillation in still water, $\mathrm{V}_{\mathrm{R}}=1 / \mathrm{f}_{\mathrm{s}}^{*}$

response function: VIV response function is a function $A^{*}=A^{*}\left(V_{R}, K_{s}\right)$ such as those defined in [1] providing the normalized VIV response amplitude $A^{*}=A / D$ as a function of reduced velocity $V_{R}=1 / f_{s}^{*}$, and the Scruton damping parameter $\mathrm{K}_{\mathrm{s}}=\pi^{2} \mathrm{~m}_{\mathrm{es}, \mathrm{avg}}{ }^{*} \zeta$. Secondary parameters (see below) may also influence response amplitude. If so, fixed given values of these secondary parameters are considered. 
secondary parameters: parameters other than reduced velocity $V_{R}$ and the Scruton mass damping parameter $K_{s}$ that may influence maximum VIV amplitude, including but not limited to mass ratio $\left(\mathrm{m}_{\text {ratio }}\right)$, mode shape $(\phi)$, Reynolds number, pipe roughness, and turbulence and/or unsteadiness of incoming flow.

Sivana simplified version of Vivana [4] introduced in [8] and formulated in Section 3

straked: strake-covered, although the general principles may be applied to any type of strake, where reference to specific quantitative results is made, it applies to strakes with 3 strands around the circumference at a pitch of $17.5 \mathrm{D}$ and a strand height of $0.25 \mathrm{D}$.

transverse: "Transverse displacement" is vertical for cross-flow VIV, and horizontal (lateral) for in-line VIV. These are considered as separate cases. Interactions between vertical and lateral motions are not considered in the formulation but included in estimating effective values of the excitation coefficient.

Vivana Frequency domain VIV response code [4,5] based on the assumption of harmonic motion and that such motion gives rise to a harmonic hydrodynamic force on the pipe, which is defined by excitation and added mass functions, and the influence of VIV amplitude on added mass is neglected.

Vivana default excitation and added mass functions: this refers to the functions defined in [4]. These functions have been updated in [5], but [5] is only available to holders of a license for the Vivana code.

\subsection{Latin Symbols}

A = amplitude of harmonic vibration at the location $\mathrm{x}$ along the span where it is a maximum

$\mathrm{A}^{*} \quad=$ normalized amplitude $\mathrm{A}$, given by $\mathrm{A}^{*}=\mathrm{A} / \mathrm{D}$, corresponds to maximum of the local amplitude $\mathrm{Y}^{*}$, which typically (but not necessarily) occurs at midspan

$\mathrm{A}^{*}\left(\mathrm{~V}_{\mathrm{R}}, \mathrm{K}_{\mathrm{s}}\right)=\mathrm{VIV}$ amplitude response function, in particular, in Section 5, this refers to F105 response function for cross-flow VIV as defined under terms above

$\mathrm{A}_{\mathrm{u}}{ }^{*}\left(\mathrm{~V}_{\mathrm{R}}\right)=$ undamped response function, $\mathrm{A}_{\mathrm{u}}{ }^{*}\left(\mathrm{~V}_{\mathrm{R}}\right)=\mathrm{A}^{*}\left(\mathrm{~V}_{\mathrm{R}}, 0\right)$

$\mathrm{C}_{\mathrm{a}}=\mathrm{C}_{\mathrm{a}}\left(\mathrm{f}^{*}\right)=$ added mass coefficient under flowing conditions. As in [4], this is assumed to be a function of dimensionless frequency $\mathrm{f}^{*}$ only, i.e. $\mathrm{C}_{\mathrm{a}}=\mathrm{C}_{\mathrm{a}}\left(\mathrm{f}^{*}\right)$.

$\mathrm{C}_{\mathrm{as}} \quad=$ added mass coefficient in still water, the values used here are $\mathrm{C}_{\mathrm{as}}=1$ for bare pipe, $\mathrm{C}_{\mathrm{as}}=1.51$ for straked pipe. For Implementation A, the straked or bare values are used, as applicable, but for Implementation B, the bare value is used on strake-covered portions of the pipe as well as bare ones. This Implementation B provision only applies for the still water added mass and not for the added mass under flowing conditions, defined above.

$\mathrm{C}_{\mathrm{e}}=\mathrm{C}_{\mathrm{e}}\left(\mathrm{f}^{*}, \mathrm{Y}^{*}\right)=$ excitation coefficient. Also referred to as "lift coefficient" for in-line VIV. The function $\mathrm{C}_{\mathrm{e}}(.,$. is the excitation function.

$\mathrm{C}_{\mathrm{en}}=\mathrm{C}_{\mathrm{en}}(\mathrm{r})=$ normalized excitation function, see Eqs. 12 and 13

$\mathrm{C}_{\mathrm{en} 2 \mathrm{D}}=\mathrm{C}_{\mathrm{en} 2 \mathrm{D}}(\mathrm{r})=$ normalized excitation function when calibrated to the response function from a $2 \mathrm{D}$ test, see Eqs. 9 and 10.

$\mathrm{D} \quad=$ pipe outer diameter including any coatings

$\mathrm{E}_{\mathrm{in}} \quad=$ net energy input into the span per cycle of harmonic motion

$\mathrm{E}_{\mathrm{in}}{ }^{*}=\mathrm{E}_{\mathrm{in}} /\left(2 \mathrm{~m}_{\mathrm{w}} \mathrm{L} \mathrm{U^{2 }}\right)=$ dimensionless energy input

EI = effective flexural stiffness of the pipe, including contribution from coatings, if applicable

$\mathrm{f} \quad=$ frequency of VIV oscillation

$\mathrm{f}^{*} \quad=$ dimensionless frequency of VIV oscillation, $\mathrm{f}^{*}=\mathrm{fD} / \mathrm{U}$

$\mathrm{f}_{\mathrm{s}} \quad=$ undamped natural frequency in still water, for Implementation A it is the natural frequency of the partially strake-covered pipe, for Implementation B it is the natural frequency of the bare pipe.

$\mathrm{f}_{\mathrm{s}}{ }^{*}=\mathrm{f}_{\mathrm{s}} \mathrm{D} / \mathrm{U}=1 / \mathrm{V}_{\mathrm{R}}=$ dimensionless, still-water, undamped natural frequency; $1 / \mathrm{f}_{\mathrm{s}}^{*}$ is the reduced velocity $V_{R}$

$\mathrm{k}=$ soil spring stiffness (force per unit length along pipe per unit deflection), refers to stiffness of vertical or horizontal springs for cross-flow or in-line VIV, respectively 
$\mathrm{K}_{\mathrm{s}}=\pi^{2} \mathrm{~m}_{\mathrm{es}, \text { avg }}{ }^{*} \zeta=$ Scruton damping/stability parameter. For implementation A the value for the partially strake-covered pipe; for Implementation B the value for the bare pipe.

$\mathrm{K}_{\mathrm{s}}() \quad=$. inverse function to $\mathrm{R}_{\mathrm{K}}($.$) defined in Eq. 8$

$\mathrm{L} \quad=$ span length or any other reference length (e.g. overall length of a zone of multiple interacting spans)

$\mathrm{m}_{\text {es,avg }}{ }^{*}=\int_{\text {span }}\left(\mathrm{m}_{\text {ratio }}+\mathrm{C}_{\mathrm{as}}\right) \phi^{2} \mathrm{dx} /\left\{\int_{\text {mode }} \phi^{2} \mathrm{dx}\right\}=\mathrm{M}_{\mathrm{es}}{ }^{*} / \phi_{\mathrm{rms}}{ }^{2}=$ modal effective mass ratio in still water; for Implementation $\mathrm{B}$, or for $100 \%$ bare or $100 \%$ straked pipe this is simply $\mathrm{m}_{\text {ratio }}+\mathrm{C}_{\mathrm{as}}$.

$\mathrm{m}_{\mathrm{p}} \quad=$ mass of pipe, coatings, and content per unit length of pipe, for Implementation A this also includes the mass of the strakes, for the straked portions, but for Implementation B, the mass of the strakes is excluded

$\mathrm{m}_{\text {ratio }}=\mathrm{m}_{\mathrm{p}} / \mathrm{m}_{\mathrm{w}}=$ mass ratio

$\mathrm{m}_{\mathrm{w}} \quad=$ mass of water displaced by the bare pipe per unit length of pipe $\left(1 / 4 \pi D^{2} \rho\right)$

$\mathrm{N} \quad=$ effective axial force in the pipeline where it spans, positive for tension

$\mathrm{N}_{\mathrm{c}} \quad$ = buckling load; absolute value of $\mathrm{N}$ at which buckling in compression occurs

$\mathrm{N}_{\mathrm{r}} \quad=$ number of points used in the discretization to solve the integral equation arising from the inversion problem

$\mathrm{r} \quad=$ ratio of damped to undamped VIV amplitude, $\mathrm{r}=\mathrm{A}^{*} / \mathrm{A}_{\mathrm{u}}{ }^{*}$

$\mathrm{R}_{\mathrm{K}}()=$. function such that $\mathrm{R}_{\mathrm{K}}\left(\mathrm{K}_{\mathrm{s}}\right)$ provides the factor by which the VIV amplitude is reduced by the damping $\mathrm{K}_{\mathrm{s}}$ according to the $\mathrm{F} 105$ cross-flow response function, defined in Eq. 7

$\mathrm{U} \quad=$ current velocity, more precisely, the magnitude of the component of current velocity normal to the pipeline at the pipeline elevation that would occur there if the pipeline were not present, assumed to be constant along the length of the span

$\mathrm{V}_{\mathrm{R}}=1 / \mathrm{f}_{\mathrm{s}}^{*}=$ reduced velocity, for partially straked pipe it is based on the natural frequency with the strakes for Implementation A, and without the strakes for Implementation B.

$\mathrm{x}=$ coordinate defining location along the pipeline

$\mathrm{Y}=\mathrm{Y}(\mathrm{x})=\mathrm{A} \phi(\mathrm{x})=$ amplitude of transverse displacement at location $\mathrm{x}$

$\mathrm{Y}^{*}=\mathrm{Y}^{*}(\mathrm{x})=\mathrm{Y} / \mathrm{D}=\mathrm{A}^{*} \phi(\mathrm{x})=$ normalized amplitude of transverse displacement at location $\mathrm{x}$

$\mathrm{y}(\mathrm{x}, \mathrm{t})=\mathrm{Y}(\mathrm{x}) \cos (\omega \mathrm{t})=\mathrm{A} \phi(\mathrm{x}) \cos (\omega \mathrm{t})=$ transverse displacement at location $\mathrm{x}$ and time $\mathrm{t}$

\subsection{Greek \& Mathematical Symbols}

$\phi=\phi(\mathrm{x})=$ still water mode shape; normalized so that $\phi(\mathrm{x})=1$ at the location $\mathrm{x}$ where the modal displacement is a maximum in absolute value; for Implementation A, this is the undamped mode shape for the partially straked pipe; for Implementation B it is the mode for the bare pipe

$\phi_{\mathrm{rms}}=$ root mean square modal displacement, $\phi_{\mathrm{rms}}{ }^{2}=\mathrm{L}^{-1} \int_{\text {mode }} \phi^{2} \mathrm{dx}$

$\rho \quad=$ density of seawater

$\omega=2 \pi \mathrm{f}=$ angular frequency of VIV oscillation (under flowing conditions)

$\omega_{\mathrm{s}}=2 \pi \mathrm{f}_{\mathrm{s}}=$ undamped natural frequency of vibration in still water; for the partially straked pipe for Implementation A, but for the bare pipe for Implementation B.

$\zeta \quad=$ modal damping ratio in still water due to soil and structural damping, not including any hydrodynamic damping; for the partially straked pipe for Implementation A, but for the bare pipe for Implementation B.

$\int_{\text {mode }}(.) \mathrm{dx}=$ integral of (.) covering the span(s) considered plus soil-supported portions of the pipe where the modal displacement is significant

$\int_{\text {span }}() d x=$. integral of (.) covering the span(s), excluding soil-supported portions of the pipe 


\section{Sivana Assumptions and Formulation}

Whereas the basic principles of Sivana and results from applying these to in-line VIV are given in [8], details of the assumptions and formulation are given in this section but not in [8]. As in [4], linear behaviour of the pipesoil system with viscous damping and harmonic response are assumed. Further, in "Sivana", a Rayleigh-Ritz approximation is used, based on the undamped, still-water vibration mode $\phi=\phi(\mathrm{x})$. The frequency is obtained from the Rayleigh quotient, and the damping from an energy balance condition.

For partially straked spans, there are two possible implementations of the Sivana approach, depending on whether the undamped, still-water modes with or without the strakes are used in the Rayleigh-Ritz approximation. These are referred to as Implementation A and B, respectively. The same equations apply for both implementations, but the definition of certain symbols differs as specified in the Nomenclature section. For instance, $\phi=\phi(\mathrm{x})$ denotes the undamped, still-water mode shape with the strakes for Implementation A and without the strakes for Implementation $B$. The dynamic properties $f_{s}, \zeta$, and $K_{s}$ apply to the pipe with strakes for implementation A, but without the strakes for Implementation B. All results presented in this paper are based on Implementation B.

As in Vivana [4], it is assumed that, at any cross section $\mathrm{x}$, for a given harmonic transverse displacement $\mathrm{Y}(\mathrm{x}) \cos (\omega \mathrm{t})$, the hydrodynamic force per unit length exerted by the water on the pipe is also harmonic and given by $F_{m} \cos (\omega t)-F_{e} \sin (\omega t)$, where $F_{m}=\omega^{2} Y_{a} m_{w}$ is the component of hydrodynamic force in-phase with the motion, and $\mathrm{F}_{\mathrm{e}}=\mathrm{C}_{\mathrm{e}} 1 / 2 \rho \mathrm{U}^{2} \mathrm{D}$ is the component $90^{\circ}$ out-of-phase with the motion. The latter provides excitation when positive or damping when negative. Here $C_{e}=C_{e}\left(f^{*}, Y^{*}\right)$ and $C_{a}=C_{a}\left(f^{*}\right)$ are the dimensionless excitation and added mass coefficients, respectively. Neglecting Reynolds number effects, it follows from dimensional analysis that these coefficients depend only on the dimensionless frequency $\mathrm{f}^{*}=\mathrm{fD} / \mathrm{U}$ and amplitude $\mathrm{Y}^{*}=\mathrm{Y} / \mathrm{D}$. A further approximation is to neglect the effect of the amplitude $\mathrm{Y}^{*}$ on the added mass coefficient $\mathrm{C}_{\mathrm{a}}$. Both these approximations made in Vivana [4] as well as here.

To extend the validity of the expression $F_{e}=C_{e} 1 / 2 \rho U^{2} D$ to negative values of $Y$, the excitation coefficient $\mathrm{C}_{\mathrm{e}}=\mathrm{C}_{\mathrm{e}}\left(\mathrm{f}^{*}, \mathrm{Y}^{*}\right)$ must be an antisymmetric function of the amplitude $\mathrm{Y}^{*}$, since a $180^{\circ}$ phase change in the motion must bring with is a corresponding $180^{\circ}$ phase change in the hydrodynamic force. Such negative values of $\mathrm{Y}$ arise where the mode $\phi=\phi(\mathrm{x})$ is negative at certain locations $\mathrm{x}$. Henceforth $\mathrm{C}_{\mathrm{e}}\left(\mathrm{f}^{*}, \mathrm{Y}^{*}\right)$ is only defined for positive values of $\mathrm{Y}^{*}$ with the understanding that for negative values $\mathrm{C}_{\mathrm{e}}=-\mathrm{C}_{\mathrm{e}}\left(\mathrm{f}^{*},\left|\mathrm{Y}^{*}\right|\right)$.

Following the Rayleigh-Ritz approach, the pipe motion is assumed to be of the form:

$y(x, t)=\phi(x) A \cos (\omega t)$

The frequency of such motion is obtained from the Rayleigh quotient, considering only the forces in-phase with the motion. This yields

$\left(\omega / \omega_{\mathrm{s}}\right)^{2}=\left(\mathrm{f} / \mathrm{f}_{\mathrm{s}}\right)^{2}=\left(\mathrm{f}^{*} / \mathrm{f}_{\mathrm{s}}^{*}\right)^{2}=\int_{\text {mode }}\left(\mathrm{m}_{\text {ratio }}+\mathrm{C}_{\mathrm{as}}\right) \phi^{2} \mathrm{dx} /\left\{\int_{\text {mode }}\left(\mathrm{m}_{\text {ratio }}+\mathrm{C}_{\mathrm{a}}\right) \phi^{2} \mathrm{dx}\right\}$

Here the subscript " $\mathrm{s}$ " indicates still-water, as opposed to flowing conditions. Thus, $\omega=2 \pi f$ is the angular frequency under flowing conditions, whereas $\omega_{\mathrm{s}}=2 \pi \mathrm{f}_{\mathrm{s}}$ is the corresponding value under still-water conditions. (See also the Nomenclature section, especially for differences in symbol definitions for Implementations A and B.)

Since $\mathrm{C}_{\mathrm{a}}=\mathrm{C}_{\mathrm{a}}\left(\mathrm{f}^{*}\right)$, Eq. (2) can be solved for the dimensionless VIV oscillation frequency $\mathrm{f}^{*}$ before the amplitude $\mathrm{A}^{*}$ is known.

For the $90^{\circ}$ out-of-phase components of force, considering the structural and soil damping (captured by the Scruton number $\left.\mathrm{K}_{\mathrm{s}}\right)^{8}$ as well as the hydrodynamic excitation or damping, the energy balance condition can be written in dimensionless form as

$\mathrm{E}_{\mathrm{in}}{ }^{*}=\mathrm{A}^{*}(1 / \mathrm{L}) \int_{\mathrm{span}} \mathrm{C}_{\mathrm{e}}\left(\mathrm{f}^{*}, \mathrm{~A}^{*} \phi\right) \phi \mathrm{dx}-4 \pi \mathrm{f}^{*} \mathrm{f}_{\mathrm{s}}^{*} \mathrm{~K}_{\mathrm{s}} \mathrm{A}^{* 2} \phi_{\mathrm{rms}}{ }^{2}$

8 To derive the energy dissipated by structural and soil damping, it has been assumed that the system in still water has classical modes. I.e. that the undamped mode shapes are orthogonal with respect to the damping matrix, as well as the stiffness and mass matrices. If that is not the case, the result still applies as a RayleighRitz approximation. This approximation is tacitly made for such cases in the guidance provided in Section 4.6 of DNVGL-RP-F114 [16] to estimate damping. 
where $\mathrm{E}_{\mathrm{in}}{ }^{*}=\mathrm{E}_{\mathrm{in}} /\left(2 \mathrm{~m}_{\mathrm{w}} \mathrm{L} \mathrm{U} \mathrm{U}^{2}\right)$ is the dimensionless energy input required to achieve energy balance, and $\phi_{\mathrm{rms}}$ is the root mean square modal displacement, as defined in the Nomenclature. Thus, the amplitude of VIV is calculated as the value of $A^{*}=A / D$ for which $E_{\text {in }}{ }^{*}$ from Eq. (3) is zero.

Alternatively, with $\mathrm{E}_{\mathrm{in}}=0$, solving Eq. (3) for $\mathrm{K}_{\mathrm{s}}$, one obtains

$\mathrm{K}_{\mathrm{s}}=(1 / \mathrm{L}) \int_{\mathrm{span}} \mathrm{C}_{\mathrm{e}}\left(\mathrm{f}^{*}, \mathrm{~A}^{*} \phi\right) \phi \mathrm{dx} /\left\{4 \pi \mathrm{f}^{*} \mathrm{f}_{\mathrm{s}}^{*} \mathrm{~A}^{*} \phi_{\mathrm{rms}}{ }^{2}\right\}$

for the damping $\mathrm{K}_{\mathrm{s}}$ for which the normalized vibration amplitude is $\mathrm{A}^{*}$.

If the assumption $\mathrm{C}_{\mathrm{a}}=\mathrm{C}_{\mathrm{a}}\left(\mathrm{f}^{*}\right)$ were relaxed, allowing $\mathrm{C}_{\mathrm{a}}=\mathrm{C}_{\mathrm{a}}\left(\mathrm{f}^{*}, \mathrm{Y}^{*}\right)$, the same equations still apply, but one would need to solve Eq. 2 and Eq. 4 simultaneously for $\mathrm{f}^{*}$ and $\mathrm{A}^{*}$, instead of solving first Eq. 2 for $\mathrm{f}^{*}$ and then Eq. 4 for $\mathrm{A}^{*}$. All results presented in this paper have been obtained for the case when the amplitude $\mathrm{Y}^{*}$ does not affect the flowing added mass coefficient $\mathrm{C}_{\mathrm{a}}$.

\section{Application to In-Line VIV}

Application of the Sivana approach to pure in-line VIV is given in [8]. Therefore only the key points are summarized here.

1) The excitation and added mass functions for bare pipe are from forced vibration tests in [7], as smoothed and interpolated in [4]. For straked pipe, they are available from recent forced vibration tests reported in [8].

2) For the pipeline spans considered, undergoing first mode response, good agreement between the Vivana and Sivana predictions of in-line VIV is obtained. This confirms that propagating wave effects that are included in Vivana but not in Sivana are not important for the cases considered, which involve first mode response.

3) Good agreement is also obtained for in-line response of bare pipe between the Sivana and the F105 response function of [1], as shown in Figure 1.

4) The Sivana results are not sensitive to the mass ratio $m_{\text {ratio }}$ or the mode shape $\phi$, provided that the Scruton Damping parameter $\mathrm{K}_{\mathrm{s}}$ does not change. (Changing $\mathrm{m}_{\text {ratio }}$ at constant damping ratio $\zeta$ means that $\mathrm{K}_{\mathrm{s}}$ also changes, and that does change VIV response amplitude.)

5) Even without soil and/or structural damping, $14 \%$ strake coverage centered at midspan is sufficient to suppress pure in-line VIV for the example considered in [8] with a mass ratio of $\mathrm{m}_{\text {ratio }}=1.23$.

For many pipeline spans, in-line fatigue damage dominates in assessments performed according to [1]. This provided the motivation in [8] to first look at in-line VIV. However, in view of the high effectiveness of even a small amount of strakes to suppress in-line VIV, longer spans become permissible, and it is necessary to extend the methodology also to cross-flow VIV, despite the challenge that arises because cross-flow VIV mostly also involves a component of cross-flow-induced in-line motion. 


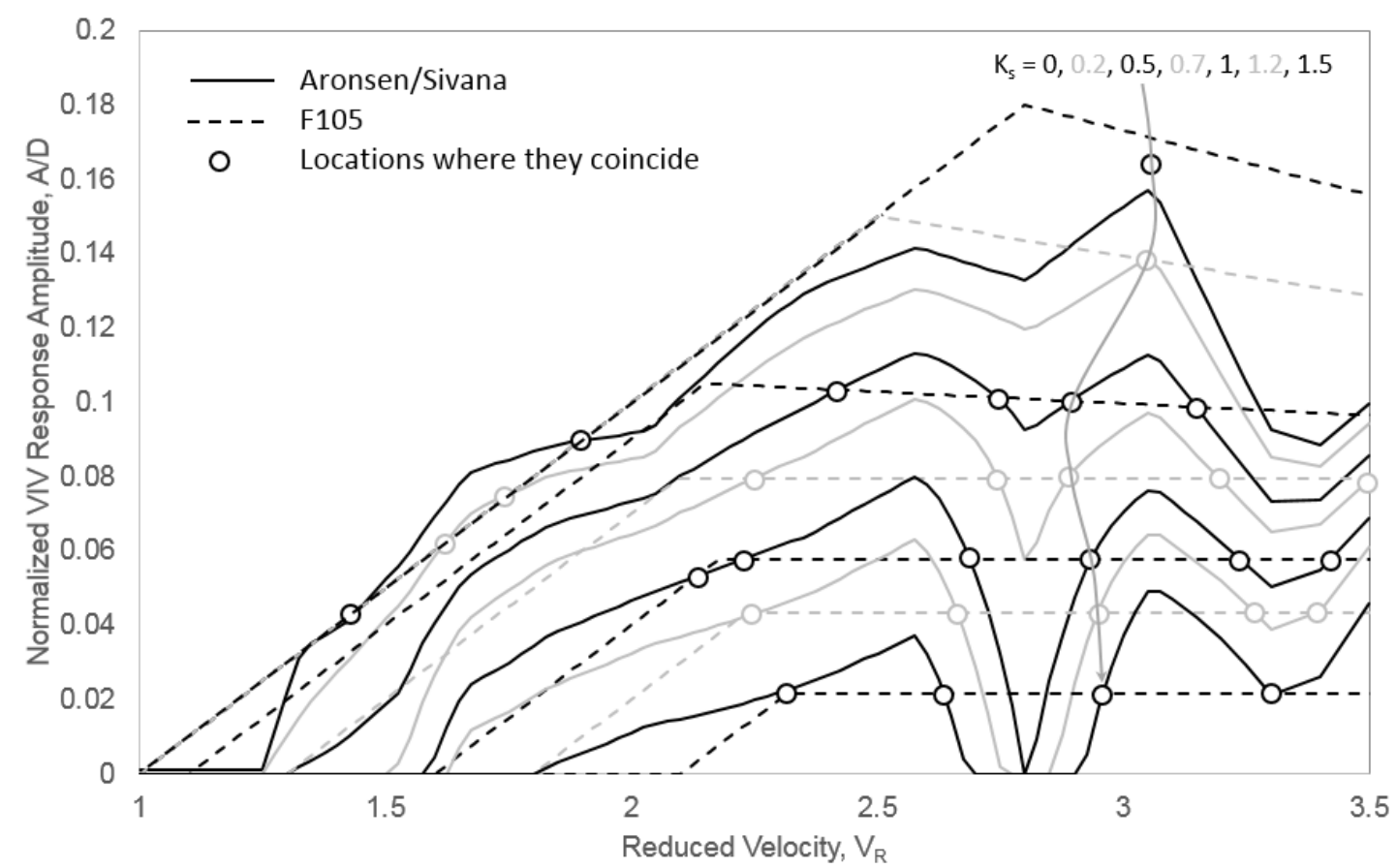

Figure 1 Comparison of F105 in-line VIV response function [1] with that predicted by Sivana using excitation and added mass functions from Aronsen [7,8], for bare pipe with mass ratio of $\mathbf{m}_{\text {ratio }}=\mathbf{1 . 2 3}$, and the mode shape from the analytical solution in $[17,18]$ for a single span with an axial force of $N / N_{c}=-\mathbf{0 . 2 0}$ and a normalized span length of $L(k / E I)^{1 / 4}=19.13$.

\section{Cross-Flow Excitation Function by Inversion of F105 Response Function}

Nothing new is needed to apply Sivana to calculate cross-flow VIV amplitudes of partially straked pipes: For straked pipe, the excitation (or "lift") and added mass functions are available from [19], and for bare pipe, they are available from forced vibration tests by Gopalkrishnan [6], with the Vivana implementation thereof described in [4].

The problem is that, for bare pipe, the above-referenced excitation and added mass functions do not give a good predictions of VIV response amplitude compared to the F105 response function from [1] that comes from VIV tests on flexible pipes, as shown in Figure 2. Possible explanations include:

1) IL-CF interaction is not considered, though CF mostly also involves a component of IL motion that is not present in the forced vibration tests from which the excitation function is derived. Such interactions are clearly evident from the efforts in [9] and [25] to determine hydrodynamic local forces and thus the excitation coefficients from the response of a well-instrumented flexible pipe. Although forced vibration tests involving in-line as well as cross-flow motion are possible, and have been performed by Aronsen [7], the results of such tests depend on a larger number of input parameters, not just on $\mathrm{f}^{*}$ and $\mathrm{Y}^{*}$. This makes it much more demanding to cover all relevant combinations of the input parameters in an experimental program of bi-directional forced vibration tests.

2) Assuming that the added mass coefficient depends only on frequency $\mathrm{f}^{*}$ and not on amplitude $\mathrm{Y}^{*}$, may be a worse approximation for cross-flow VIV than for in-line. The Vivana default added mass function corresponds to Gopalkrishnan's results at an amplitude of $\mathrm{Y}^{*}=0.5$. Picking a different amplitude or including the effect of amplitude in the added mass function would lead to different results. Amplitudes below $\mathrm{Y}^{*}=0.15$ are not captured in Gopalkrishnan's data, and these could involve very high added mass, especially near the Strouhal frequency.

3) In addition to interaction between vertical and horizontal motions, interactions with higher harmonics and higher modes could be significant [26].

4) VIV motion is not harmonic as assumed. Even a narrow-band motion can be significantly different, exhibiting beating for instance. Forced vibration tests for beating motions in [6] show that this has a significant effect on hydrodynamic forces. This is more so for cross-flow, where both the Strouhal frequency and the pipe natural frequency play a role. 
For these reasons, the excitation function from forced vibration tests in [6] have been adjusted in [4] to get the Vivana default functions used for Figure 2 [27], [24]. The recent development in the excitation functions and the response frequency model focus on multi-frequency/mode riser responses [28]. Modifications to the existing hydrodynamic load models, including both the excitation and the added mass functions, are needed to improve the VIV response prediction for free span pipelines.

Here a different calibration of the excitation function is developed, to match pipeline response as predicted by the F105 response function in [1]. This is done by mathematically inverting the Sivana analysis to go from response function to excitation function, rather than vice-versa. I.e., the excitation function is constructed to make the differences between grey and black lines in Figure 2 vanish.

The inversion process involves two steps: First the added mass function $C_{a}=C_{a}\left(f^{*}\right)$ is constructed considering the response frequencies from VIV tests on flexible pipes as well as the forced vibration test results from $[4,6]$ (Section 5.1). Then, the excitation function $\mathrm{C}_{\mathrm{e}}=\mathrm{C}_{\mathrm{e}}\left(\mathrm{f}^{*}, \mathrm{Y}^{*}\right)$ is constructed to match the F105 cross-flow response function from [1] (Section 5.2).

There is a complication: According to Sivana, the VIV amplitude depends not only on the reduced velocity $\mathrm{V}_{\mathrm{R}}=1 / \mathrm{f}_{\mathrm{s}}^{*}$ and the Scruton Damping parameter $\mathrm{K}_{\mathrm{s}}$, but also on the mass ratio $\mathrm{m}_{\text {ratio }}$ and the mode shape $\phi=\phi(\mathrm{x})$, yet these effects are not reflected in the F105 response function. To address this, the inversion is performed for the calibration-basis parameters shown in Table 1, which are chosen to be representative of the conditions for which the F105 response function is calibrated. The same excitation function is then also used for other values of the mass ratio and mode shape.

This inversion approach is applied only for the bare pipe. For any straked segments, the excitation and added mass functions available in [19] are used.

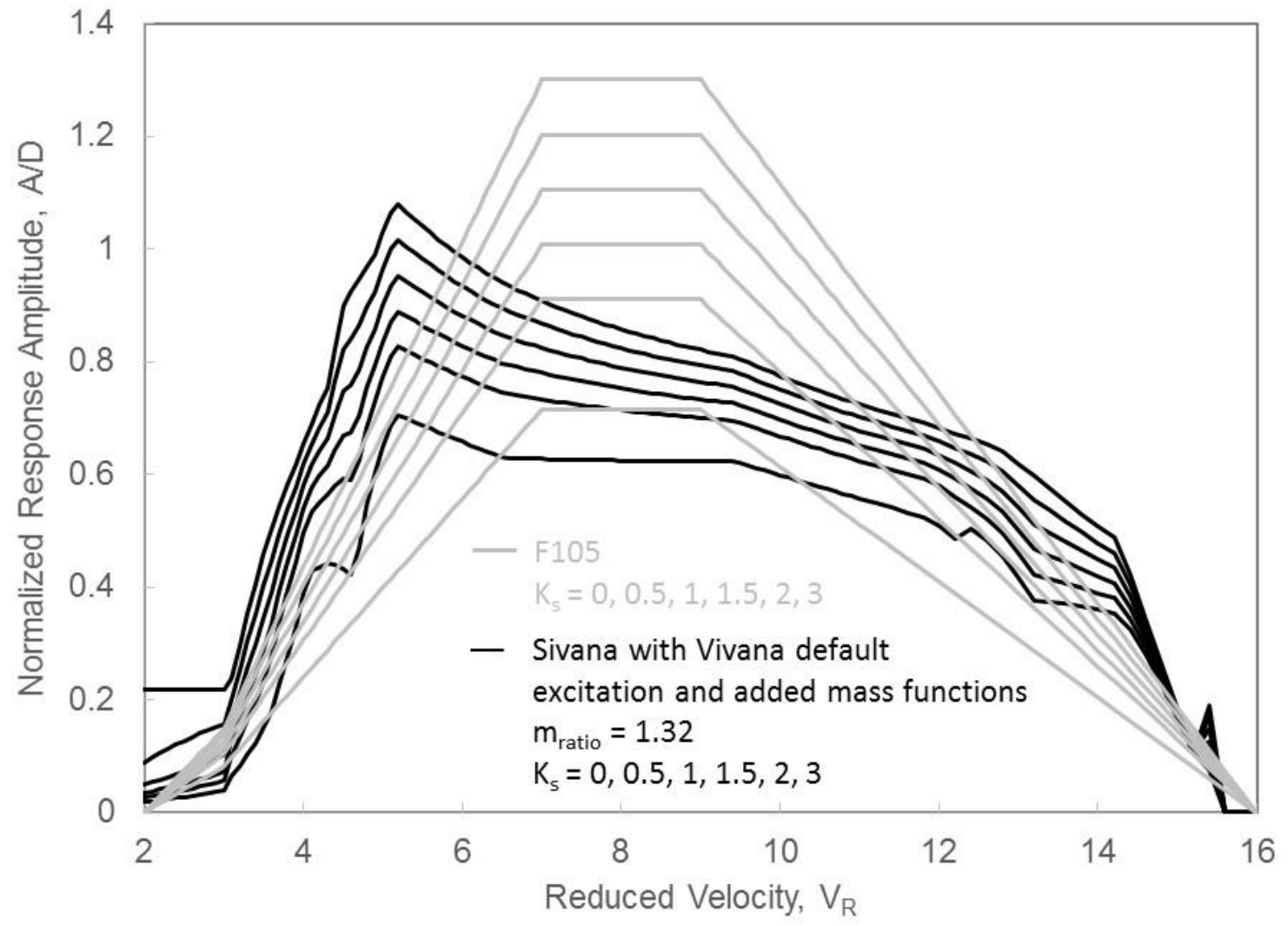

Figure 2 Cross-flow response function $A^{*}=A^{*}\left(V_{R}, K_{s}\right)$ from Sivana using Vivana default excitation and added mass functions compared with F105 cross-flow response function. (This Sivana response function is calculated for the mass ratio and mode shape of Table 1. For F105, the same response function applies for a range of mass ratios $m_{\text {ratio }}$ and mode shapes $\phi$.) 


\begin{tabular}{|l|l|l|}
\hline Description & Symbol & Value \\
\hline Mass Ratio & $\mathrm{m}_{\text {ratio }}$ & 1.32 \\
\hline Mode Shape & $\phi=\phi(\mathrm{x})$ & $\begin{array}{l}\text { Determined from analytical solution for a single span from [17,18] } \\
\text { with } \mathrm{N} /(\mathrm{k} \mathrm{EI})^{1 / 2}=-0.01508 \text { and } \mathrm{L}(\mathrm{k} / \mathrm{EI})^{1 / 4}=27.47 . \text { Plotted in Figure } \\
8 .\end{array}$ \\
\hline
\end{tabular}

Table 1 Cross-Flow calibration-basis parameters for which the excitation function is calibrated to return the F105 response function

\subsection{Bare Pipe Cross-Flow Added Mass Function}

For bare pipe, both $\mathrm{C}_{\mathrm{a}}$ and $\mathrm{C}_{\mathrm{as}}$ are constant with respect to location $\mathrm{x}$ along the span, so that Eq. 2 reduces to

$\left(\mathrm{f}^{*} / \mathrm{f}_{\mathrm{s}}^{*}\right)^{2}=\left(\mathrm{m}_{\text {ratio }}+\mathrm{C}_{\mathrm{as}}\right) /\left(\mathrm{m}_{\text {ratio }}+\mathrm{C}_{\mathrm{a}}\right)$

Measuring the frequencies from tests on flexible pipes in [20] and calculating the corresponding $\mathrm{C}_{\mathrm{a}}$ using Eq. 5, leads to Figure 3. Also shown is the added mass function recommended in [1] to count cycles of fatigue damage. This is expressed as a function of reduced velocity $V_{R}=1 / f_{s}^{*}$. This will be referred to as the "F105 added mass function". Using Eq. 5 to transform the F105 added mass function of Figure 3 to the form $C_{a}=C_{a}\left(f^{*}\right)$ leads to Figure 4. For comparison, the Vivana default added mass function from [4] is also included.

Observations and remarks about this plot are:

1) The F105 added mass function depends on the mass ratio. It should not. At least, the results of a forced vibration test should not depend on the mass ratio. For a given imposed motion of the cylinder, the mass of the pipe will influence the total force that needs to be applied to the cylinder to impose the motion, but it does not influence the hydrodynamic force.

2) The F105 relation between $\mathrm{f}^{*}$ and $\mathrm{C}_{\mathrm{a}}$ exhibits looping behavior. I.e., between points $\mathrm{D}$ and $\mathrm{E}$ Figure 4 , $C_{a}=C_{a}\left(f^{*}\right)$ is multiple-valued. I.e. there is a relationship between $C_{a}$ and $f^{*}$, but $C_{a}$ is not a function of $f^{*}$. A forced vibration test at a given $\left(f^{*}, Y^{*}\right)$, should yield just one $C_{a}$ value. However, a Sivana type model with $\mathrm{C}_{\mathrm{a}}=\mathrm{C}_{\mathrm{a}}\left(\mathrm{f}^{*}, \mathrm{Y}^{*}\right)$ could explain why the apparent added mass functions obtained from tests on a flexible pipe, or a flexibly supported rigid cylinder exhibits looping behavior.

3) In the mapping from Figure 3 to Figure 4, labelled points A to F serves to indicate which points map to where. At point $\mathrm{D}$ the dimensionless frequency $\mathrm{f}^{*}$ is a minimum. This occurs at a reduced velocity of about 6. At higher current velocities (from D towards $E$ ), the increase in oscillation frequency is less than proportional to the increase in current velocity, according to the F105 added mass function.

The above observations do call for further investigation and suggest that enhanced versions of Vivana and/or Sivana that allow $C_{a}=C_{a}\left(f^{*}, Y^{*}\right)$ instead of being limited to $C_{a}=C_{a}\left(f^{*}\right)$ may be appropriate. However, the objective here is not to revisit the effort that went into the development of the F105 response and added mass functions from VIV test data, but rather to build on those results, in order to extend the F105 guidance, at least in the interim, for partially straked pipes, sticking with the Vivana/Sivana assumption that the added mass is a function of dimensionless frequency only. For that purpose, the added mass function shown by the heavy black line in Figure 4 is used in what follows. It agrees with the F105 function for the calibration-basis mass ratio up to normalized velocities $\left(1 / \mathrm{f}^{*}\right)$ of around 6 , and then transitions steeply to the Vivana default function. 


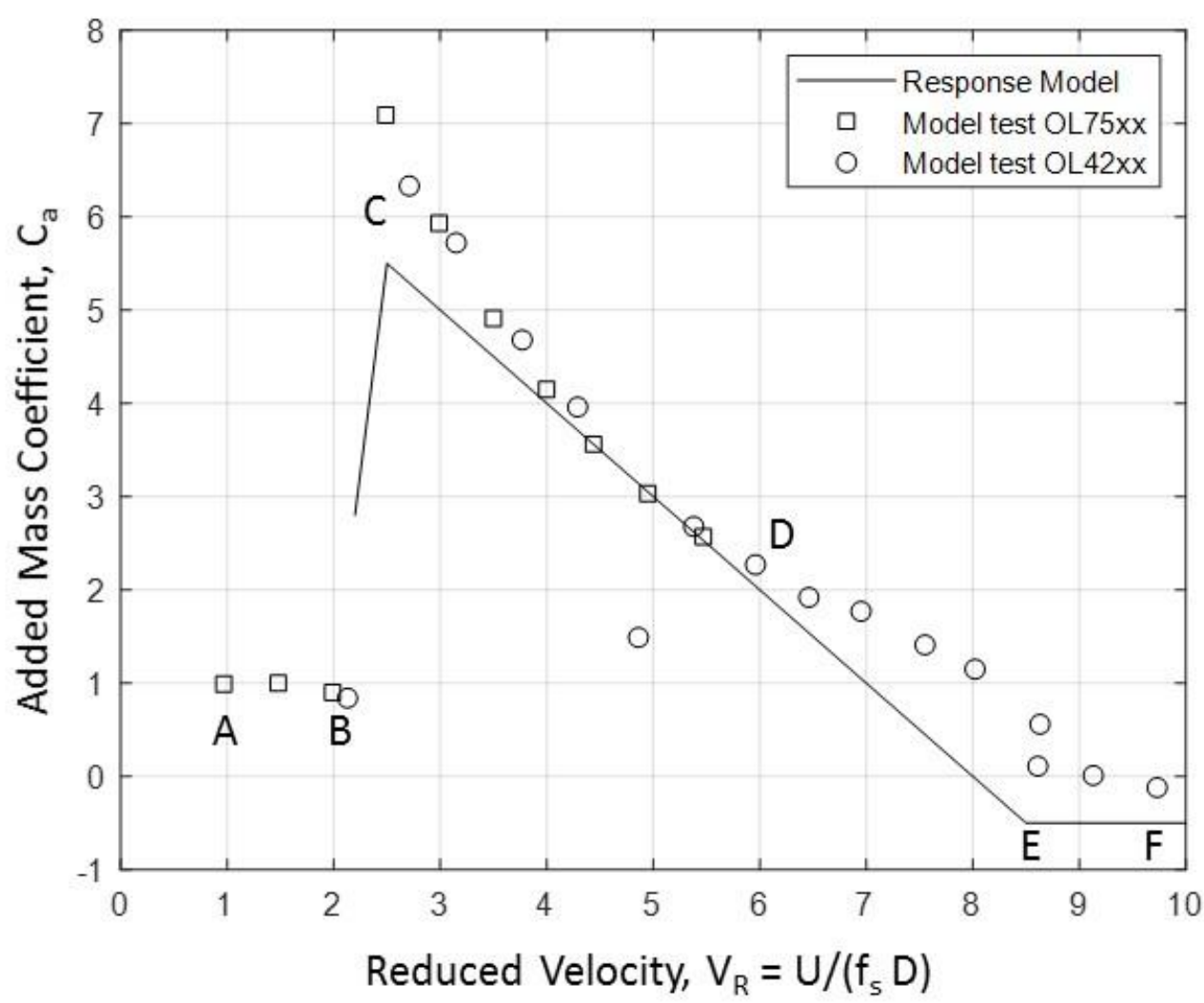

Figure 3 F105 added mass function from [1] compared with added mass calculated from response frequencies in VIV tests on flexible pipes in [20], with a mass ratio of $m_{\text {ratio }}=1.3$ for test series " $75 \times x$ " and $m_{\text {ratio }}=1.3$ for test series " $42 \mathrm{xx}$ ".

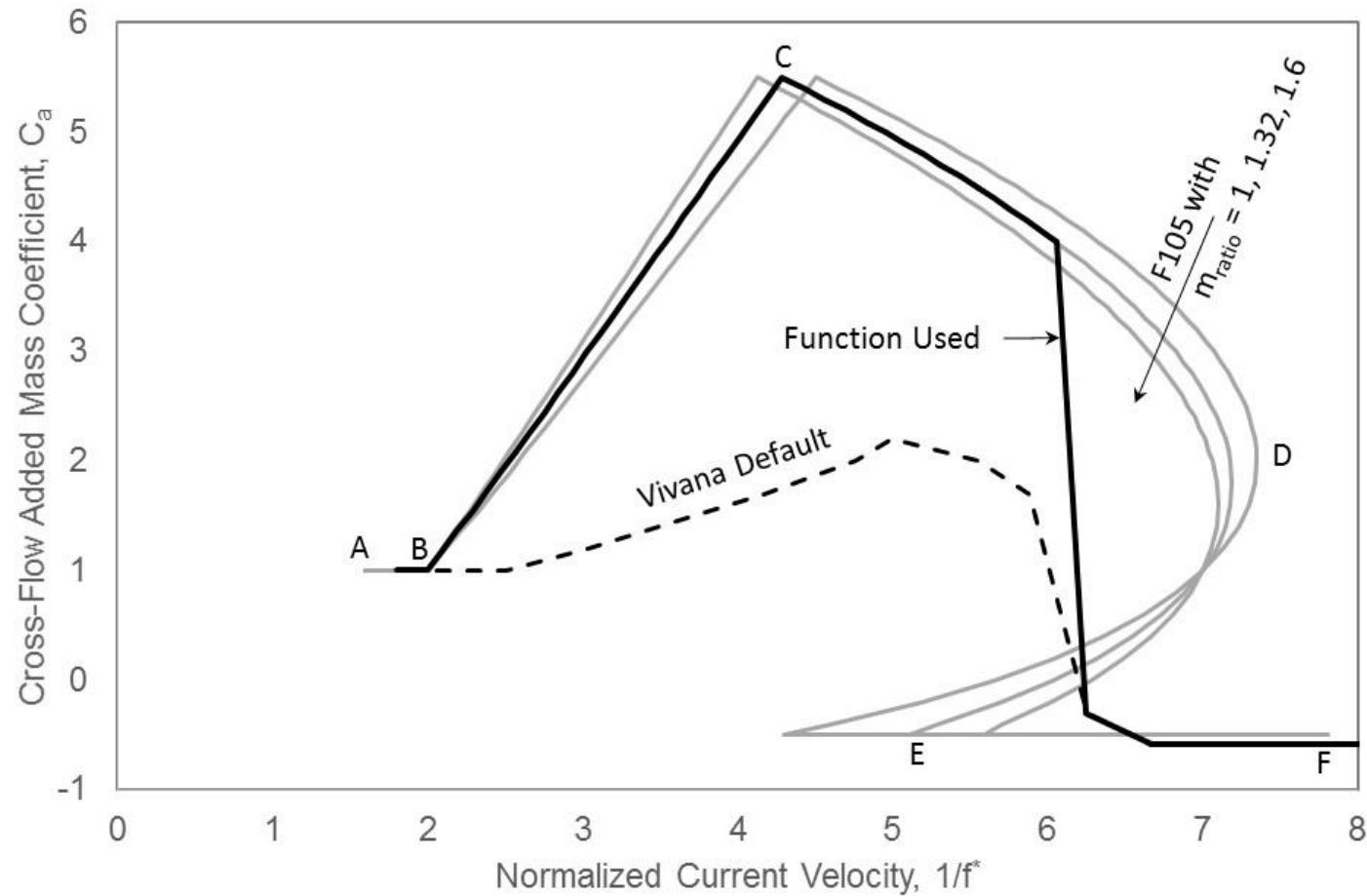

Figure 4 Cross-flow added mass function from F105 [1] (grey lines) compared with Vivana default [4] and chosen added mass function used here 


\subsection{Bare Pipe Cross-Flow Excitation Function}

The section presents the inversion process to get the cross-flow excitation function $C_{e}=C_{e}\left(f^{*}, Y^{*}\right)$ for which the F105 response function is matched when using Sivana with the calibration basis mass ratio $\mathrm{m}_{\text {ratio }}$ and mode shape $\phi=\phi(\mathrm{x})$ of Table 1. All calculations described in this section are done for the calibration-basis parameters of Table 1, even if the resulting excitation function is to be applied for a different mass ratio and/or mode shape. The F105 cross-flow response function can be written as

$A^{*}\left(V_{R}, K_{s}\right)=A_{u}^{*}\left(V_{R}\right) R_{k}\left(K_{s}\right)$

where $A_{u}{ }^{*}\left(V_{R}\right)=A^{*}\left(V_{R}, 0\right)$ is a piecewise linear function defined in F105 and shown in "Figure 4-2" of [1], that gives the undamped $\left(\mathrm{K}_{\mathrm{s}}=0\right)$ response amplitude, and

$$
\begin{aligned}
\mathrm{R}_{\mathrm{K}}\left(\mathrm{K}_{\mathrm{s}}\right) & =1-0.15 \mathrm{~K}_{\mathrm{s}} & & \text { for } \mathrm{K}_{\mathrm{s}} \in[0,4] \\
& =3.2 / \mathrm{K}_{\mathrm{s}}^{1.5} & & \text { for } \mathrm{K}_{\mathrm{s}} \in[4, \infty)
\end{aligned}
$$

$\mathrm{R}_{\mathrm{K}}($.$) is a monotonic function. The corresponding inverse function \mathrm{K}_{\mathrm{s}}($.$) given by$

$$
\begin{aligned}
\mathrm{K}_{\mathrm{s}}(\mathrm{r}) & =(3.2 / \mathrm{r})^{2 / 3} & & \text { for } \mathrm{r} \in(0,0.4] \\
& =(1-\mathrm{r}) / 0.15 & & \text { for } \mathrm{r} \in[0.4,1]
\end{aligned}
$$

The Sivana-based inversion to get the excitation function from the response function is particularly simple for a $2 \mathrm{D}$ test. This refers to a test involving a spring-supported rigid cylinder, where the mode shape is $\phi(\mathrm{x})=1 \forall \mathrm{x}$. For this case, Eq. 4 yields directly

$$
\mathrm{C}_{\mathrm{e}, 2 \mathrm{D}}\left(\mathrm{f}^{*}, \mathrm{~A}^{*}\right)=4 \pi \mathrm{f}^{*} \mathrm{f}_{\mathrm{s}}^{*} \mathrm{~A}_{\mathrm{u}}{ }^{*} \mathrm{C}_{\mathrm{en} 2 \mathrm{D}}\left(\mathrm{A}^{*} / \mathrm{A}_{\mathrm{u}}{ }^{*}\right)
$$

where $\mathrm{C}_{\mathrm{en} 2 \mathrm{D}}(\mathrm{r})=\mathrm{r} \mathrm{K}_{\mathrm{s}}(\mathrm{r})$ is referred to as the normalized excitation function, plotted in Figure 5, and given by

$$
\begin{aligned}
& \mathrm{C}_{\mathrm{en2D}}(\mathrm{r})=(10.24 \mathrm{r})^{1 / 3} \quad \text { for } r \in(0,0.4] \\
& \mathrm{C}_{\text {en2D }}(\mathrm{r})=\mathrm{r}(1-\mathrm{r}) / 0.15 \quad \text { for } \mathrm{r} \in[0.4,1]
\end{aligned}
$$

To evaluate $\mathrm{C}_{\mathrm{e}, 2 \mathrm{D}}\left(\mathrm{f}^{*}, \mathrm{~A}^{*}\right)$, write Eq. 5 as,

$\mathrm{f}_{\mathrm{s}}^{*}=\mathrm{f}^{*}\left[\left(\mathrm{~m}_{\text {ratio }}+\mathrm{C}_{\mathrm{a}}\left(\mathrm{f}^{*}\right)\right) /\left(\mathrm{m}_{\text {ratio }}+\mathrm{C}_{\mathrm{as}}\right)\right]^{1 / 2}$

and use it to calculate $\mathrm{f}_{\mathrm{s}}{ }^{*}$ and $\mathrm{V}_{\mathrm{R}}=1 / \mathrm{f}_{\mathrm{s}}{ }^{*}$, then obtain the corresponding undamped response amplitude $\mathrm{A}_{\mathrm{u}}{ }^{*}=\mathrm{A}_{\mathrm{u}}{ }^{*}\left(\mathrm{~V}_{\mathrm{R}}\right)$ from the F105 undamped cross-flow response function, then $\mathrm{C}_{\mathrm{e}, 2 \mathrm{D}}\left(\mathrm{f}^{*}, \mathrm{~A}^{*}\right)$ from Eq. 9.

More generally, considering the mode shape $\phi=\phi(\mathrm{x})$ for a flexible pipe, inversion requires solving an integral equation that arises from the energy balance condition, Eq. 3. In what follows only the results are given. They can readily be verified, by substituting back into Eq. 3 to check that it is satisfied. (That is easier than finding the solution when one does not know it.) The result is

$\mathrm{C}_{\mathrm{e}}\left(\mathrm{f}^{*}, \mathrm{Y}^{*}\right)=4 \pi \mathrm{f}^{*} \mathrm{f}_{\mathrm{s}}^{*} \mathrm{~A}_{\mathrm{u}}{ }^{*} \mathrm{C}_{\mathrm{en}}\left(\mathrm{Y}^{*} / \mathrm{A}_{\mathrm{u}}{ }^{*}\right)$

where the function $\mathrm{C}_{\mathrm{en}}($.$) is the solution to the following integral equation$

$\left(1 /\left(\mathrm{L} \phi_{\mathrm{rms}}^{2}\right)\right) \int_{\mathrm{span}} \mathrm{C}_{\mathrm{en}}(\mathrm{r} \phi) \phi \mathrm{dx}=\mathrm{C}_{\mathrm{en} 2 \mathrm{D}}(\mathrm{r}) \quad \forall \mathrm{r} \in[0,1]$

To evaluate $C_{e}\left(f^{*}, Y^{*}\right)$, proceed in the same way as for $C_{e 2 D}\left(f^{*}, A^{*}\right)$ : use Eq. 11 to obtain $f_{s}^{*}$ and $V_{R}=1 / f_{s}^{*}$, then $\mathrm{A}_{\mathrm{u}}{ }^{*}=\mathrm{A}_{\mathrm{u}}{ }^{*}\left(\mathrm{~V}_{\mathrm{R}}\right)$ from $\mathrm{F} 105[1]$, and finally $\mathrm{C}_{\mathrm{e}}\left(\mathrm{f}^{*}, \mathrm{Y}^{*}\right)$ from Eq. 12.

The integral equation, Eq. 13, needs to be solved only once for the calibration-basis mode shape. For $r \leq 0.4$, the solution is

$\mathrm{C}_{\mathrm{en}}(\mathrm{r})=\mathrm{C}_{\mathrm{en} 2 \mathrm{D}}(\mathrm{r}) / \Phi_{1} \quad$ for $\mathrm{r} \in[0,0.4]$

where

$$
\begin{aligned}
\Phi_{1} & =\left[\int_{\text {span }}|\phi|^{4 / 3} \mathrm{dx}\right] /\left[\mathrm{L} \phi_{\mathrm{rms}}{ }^{2}\right] \\
& =1.1835 \text { for the calibration-basis mode shape }
\end{aligned}
$$

For $r>0.4$, the integral equation is solved numerically, by a collocation method as follows:

1) A parametrized trail function $C_{e n}(r)$ is defined by Eq. 14 for $r \leq 0.4$, and for $r>0.4, C_{e n}(r)$ it is assumed to be piecewise linear and continuous function of $r$ between evenly-spaced points at $r=r_{i}=0.4+0.6 i / N_{r}$ for $\mathrm{i}=0,1,2, \ldots, \mathrm{N}_{\mathrm{r}}$. Thus the problem of determining a continuous function $\mathrm{C}_{\mathrm{en}}(\mathrm{r})$ is reduced to determining a finite number of unknowns $C_{e n, i}=C_{e n}\left(r_{i}\right)$ for $i=1,2, \ldots, N_{r}$. 
2) The values of $C_{e n, i}$ for $i=1,2, \ldots, N_{r}$ are obtained by enforcing Eq. 13 at $r=r_{i}$ for the same range of $i$ values. This can be done sequentially for one unknown $\mathrm{C}_{\mathrm{en}, \mathrm{i}}$ at the time, as follows:

a) Suppose the solution is available up to $C_{e n, i-1}$. (Initially, for $i=1$, this is the case from Eq. 14.)

b) Substitute $r=r_{i}$ into Eq. 13. Since the integral does not extend beyond $r_{i}$, this equation involves a single unknown, $\mathrm{C}_{\mathrm{en}, \mathrm{i}}$.

c) Take the unknown $\mathrm{C}_{\mathrm{en}, \mathrm{i}}$ outside the integral. Calculate the remaining 2 integrals numerically. Solve the remaining equation for $\mathrm{C}_{\mathrm{en}, \mathrm{i}}$.

d) Repeat this for $\mathrm{i}=1,2, \ldots, \mathrm{N}_{\mathrm{r}}$ to complete the solution for $0.4<\mathrm{r} \leq 1$.

To check for convergence, this has been done for $\mathrm{N}_{\mathrm{r}}=12,24$, and 48 . The results could not be distinguished on a plot, so finally only the $\mathrm{N}_{\mathrm{r}}=48$ plot is included in Figure 5. This suggests the solution is accurate, even if it does not prove convergence of the numerical solution scheme. (Indeed for very large $\mathrm{N}_{\mathrm{r}}$ the scheme was found to diverge, probably because of the approximate numerical evaluation of the integrals. This remained limited even as the mesh for the trial functions for $\mathrm{C}_{\mathrm{en}}(\mathrm{r})$ was refined. Thus, for the numerical integration, the solution can become non-unique above a limiting $\mathrm{N}_{\mathrm{r}}$ value.)

The shape of $C_{e n}(r)$ in Figure 5 is similar to that for the $2 \mathrm{D}$ excitation function $C_{e n 2 D}(r)$, but there are quantitative differences: the peak value is down from $\mathrm{C}_{\mathrm{en} 2 \mathrm{D} @ \max }=5 / 3=1.667$ to $\mathrm{C}_{\mathrm{en} @ \max }=1.366$. The location of the peak decreases from $r=0.5$ for the $2 \mathrm{D}$ case to approximately ${ }^{9} \mathrm{r}=0.434$. The value of $\mathrm{r}$ where $\mathrm{C}_{\mathrm{en}}=0$ is down from 1 for the $2 \mathrm{D}$ case to 0.8354 . This means that, for the calibration-basis mode shape, the undamped amplitude is 1.197 times that of a $2 \mathrm{D}$ test.

For the calibration basis mode, there is never a need to evaluate $\mathrm{C}_{\mathrm{en}}($.) for $\mathrm{r}>1$. Adding strakes only reduces the $r$ value. However, for a different mode shape, Sivana could encounter $r>1$ values. Even for the calibration basis mode $r>1$ could occur during the Sivana iterative solution process while solving for the unknown vibration amplitude. For such cases, linear extrapolation from the last two points (at $r=1$, and $r=1-\Delta r$ ) is used.

Contour plots of the resulting excitation function are shown in Figure 6. It is clear that the steep transition zone in the added mass function introduced in Figure 4 has a strong impact on the excitation function that goes with it to match the F105 response function. (The transition zone is indicated in Figure 6 by dashed lines, and covers the range $0.16 \leq \mathrm{f}^{*} \leq 0.165$ in Figure 6 which corresponds to $6.06 \leq 1 / \mathrm{f}^{*} \leq 6.25$ in Figure 4 .)

9 This approximate location of the peak is obtained by fitting a parabola to the numerically calculated points at $\mathrm{r}=0.425,0.4375$, and 0.45 . 


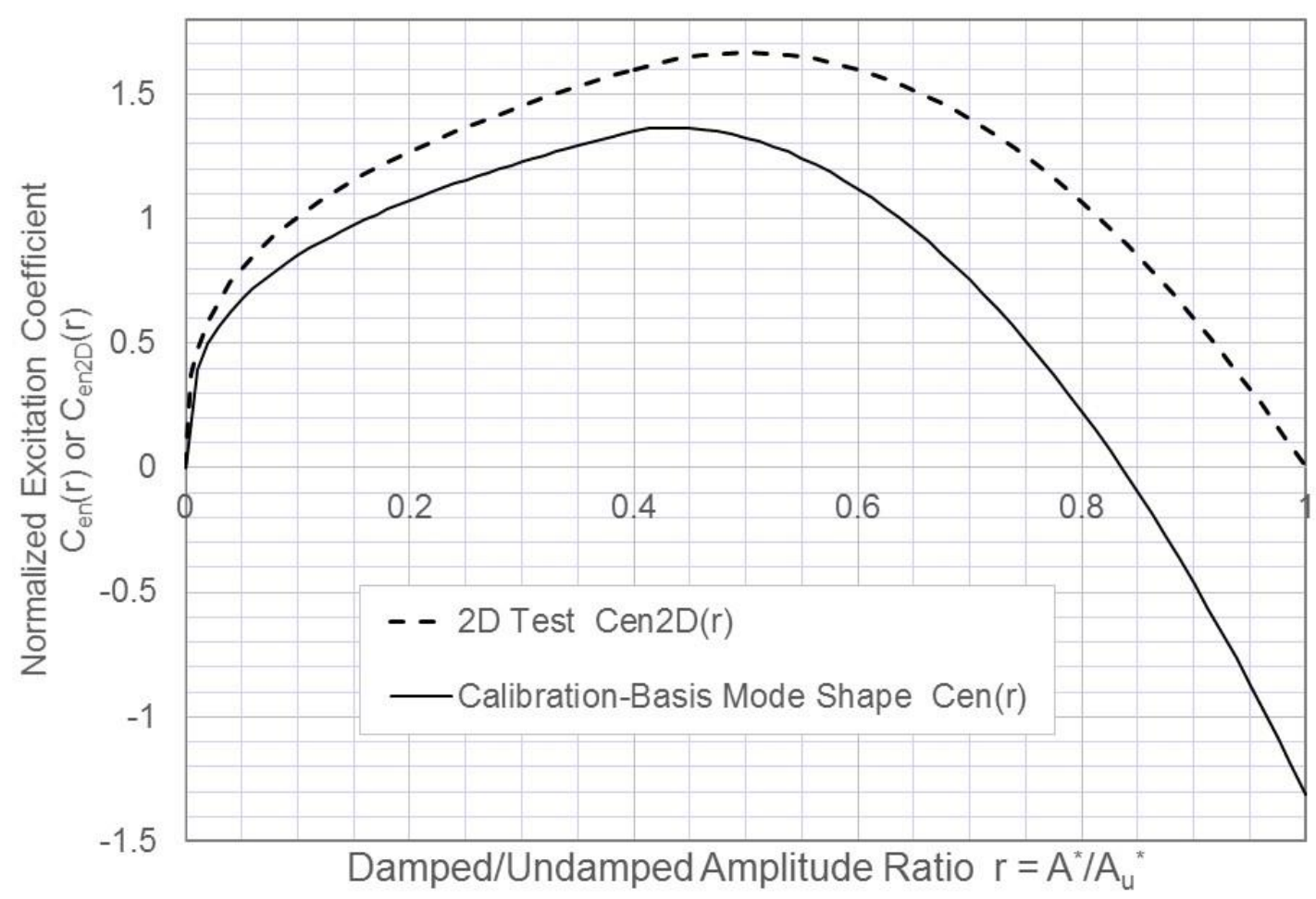

Figure 5 Normalized cross-flow excitation functions for which Sivana matches the F105 response function for the calibration-basis mass ratio and mode shape of Table 1 (continuous line). The dashed line gives the same for the mode shape of a 2D test from Eq. 10 instead of the calibration basis mode shape. 

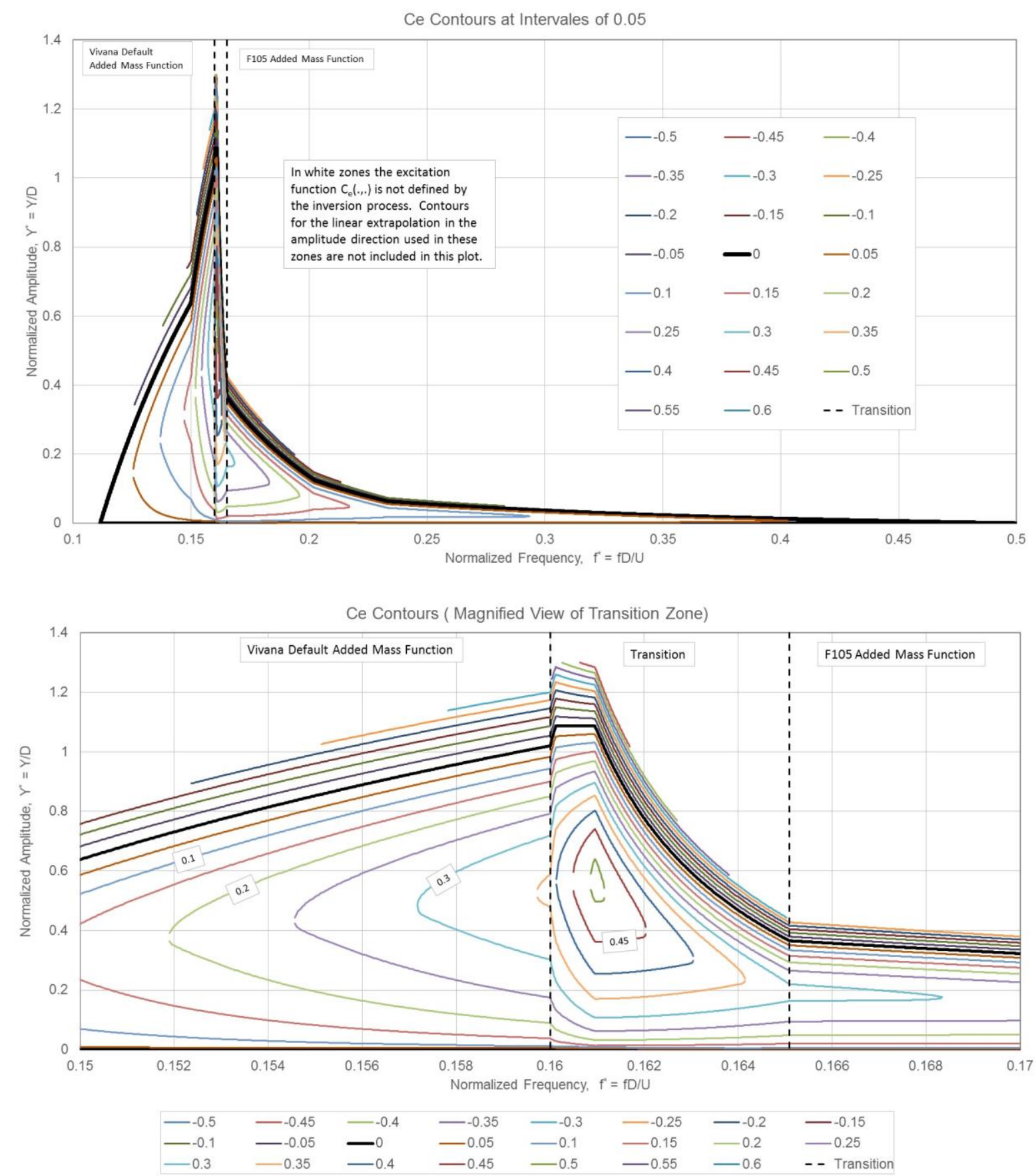

Figure 6 Contour plots of excitation function $\mathrm{C}_{e}=\mathrm{C}_{\mathrm{e}}\left(\mathbf{f}^{*}, \mathrm{Y}^{*}\right)$ for Cross-flow VIV constructed to match the F105 response function for the calibration basis mass ratio and mode shape, and the added mass function of Figure 4. (Lower shows the transition zone of the added mass function at a magnified scale.)

\section{Applications to Cross-Flow VIV}

\subsection{Bare Pipe}

By construction, applying Sivana with the calibration-basis mass ratio and mode shape of Table 1, the F105 response function is reproduced exactly. However, for Sivana the mass ratio does have some effect on the response as shown in Figure 7. The effect is only significant at high reduced velocities, beyond the response function plateau. Although this mass ratio effect is not included in [1], experiments covering a wide range of mass ratios, as in [21], do show a significant effect of the mass ratio on the bandwidth of the response function. 


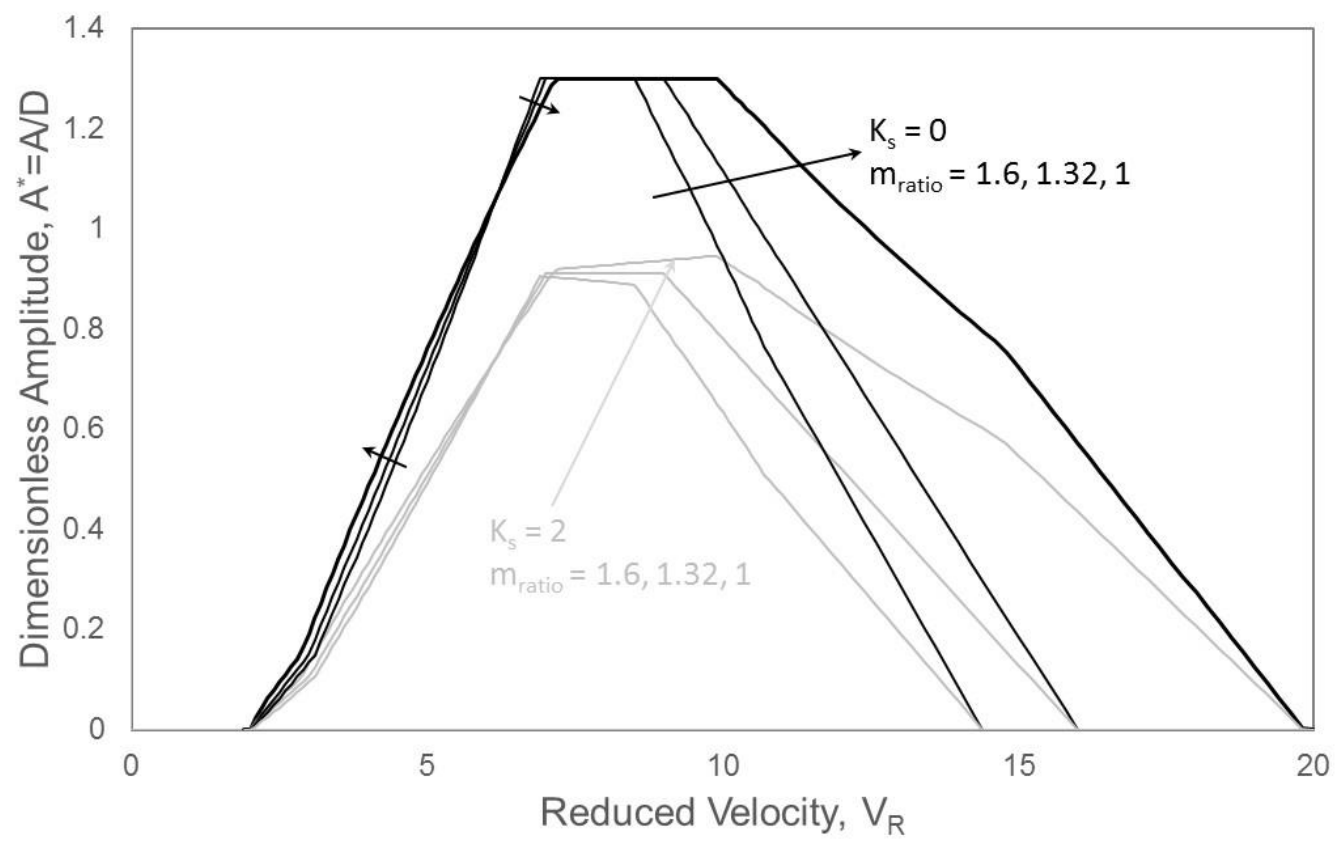

Figure 7 Sensitivity to the cross-flow VIV amplitude from Sivana to the mass ratio for values of the Scruton mass damping parameter of $K_{S}=0$ (in black) and $K_{S}=2$ (in grey). Mass ratios are 1.6, 1.32, and 1, decreasing in the direction of the arrows. For the calibration-basis mass ratio of 1.32 the result agrees with the F105 response function, as it should.

To also examine the sensitivity of the Sivana results to the mode shape, the response functions are calculated for the mode shapes shown in Figure 8. It was found that the effect of the mode shape does not depend on reduced velocity. I.e., the mode-shape effect is accounted for by a factor on amplitude that does not depend on the reduced velocity. Values of this factor are shown in Table 2 for two values of the Scruton mass damping parameter $K_{S}$. This shows that the mode shape effect is small in the range between the simply-supported and fixed-fixed mode, which should encompass most single-span cases in practice. The maximum amplitude is between 1.18 and 1.20 times that for a $2 \mathrm{D}$ test. $^{10}$

10 This refers to a 2D test simulated with Sivana using the excitation function derived from the calibration basis mode shape of Table 1 and Figure 8. 


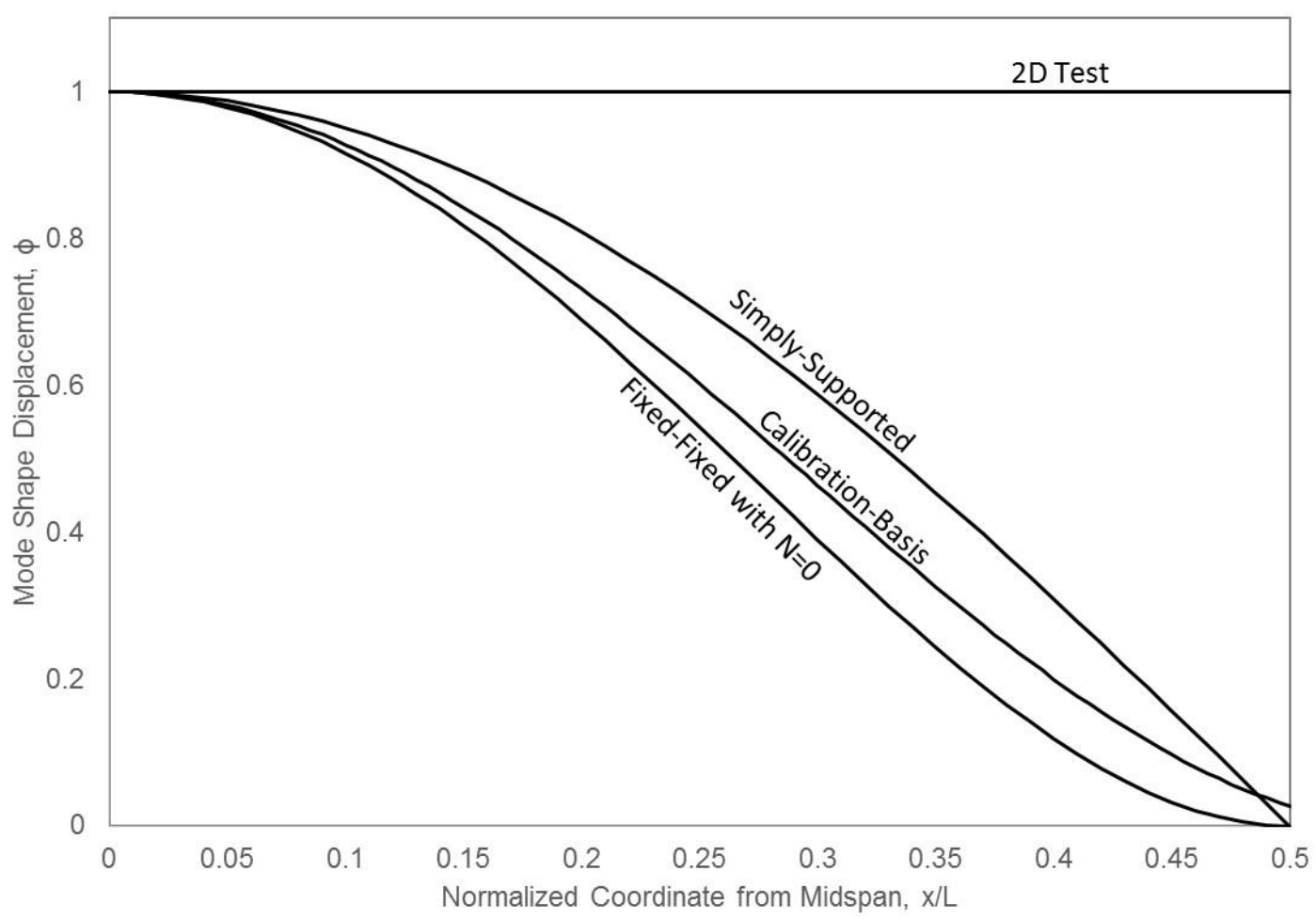

Figure 8 Mode shapes considered for mode shape sensitivity analysis, top to bottom: (1) a 2D test in which the displacement is constant along the pipe, (2) simply-supported conditions for which the mode shape is sinusoidal, (3) calibration-basis mode shape of Table 1 , and (4) the mode shape for a pipe that is fully fixed at both ends, with zero effective axial force

\begin{tabular}{|l|c|c|}
\hline & $\mathrm{K}_{\mathrm{S}}=0$ & $\mathrm{~K}_{\mathrm{S}}=2$ \\
\hline Simply-Supported & 1.178 & 1.179 \\
\hline Calibration-Basis & 1.197 & 1.200 \\
\hline Fixed-Fixed & 1.195 & 1.199 \\
\hline
\end{tabular}

Table 2 Factors to be applied to the amplitude from a 2D test ${ }^{10}$ to get the maximum amplitude for each of the mode shapes of Figure 8 for values of the Scruton damping parameter $K_{s}$ shown. These factors do not depend on the reduced velocity.

\subsection{Partially Straked Pipe}

Applying Sivana for a partially straked span with the strake coverage centred at midspan gives the results in Figure 9 for various levels of strake coverage. Structural and soil damping is assumed to be zero $\left(\mathrm{K}_{\mathrm{S}}=0\right)$, and the mass ratio and mode shape are the calibration-basis ones of Table 1 . The reduced velocity shown is that for the bare pipe. With the strakes, the natural frequency in still water is a bit lower so the reduced velocity is a bit higher. This contributes to the apparent shift in the reduced velocity at which the largest VIV amplitudes occur in Figure 9. Figure 10 shows similar results for $\mathrm{K}_{S}=2$ as Figure 9 shows for $\mathrm{K}_{\mathrm{S}}=0$. There is a general lowering of the response due to the increased non-hydrodynamic damping.

It is clear that the benefit of strakes for cross-flow is less than for the in-line case, especially on the left side of the response function. E.g. 14\% strake coverage fully eliminates in-line VIV for the example in [8], but for cross-flow, the response is only reduced by about $40 \%$ at a reduced velocity of $\mathrm{V}_{\mathrm{R}}=4$ increasing to about $50 \%$ at $V_{R}=6$. That strakes produce more reduction in cross-flow response in the left side of the response function than on the right side is in part because the strakes affect the natural frequency, and the reduced velocity shown in the plot is based on the natural frequency of the bare pipe. 


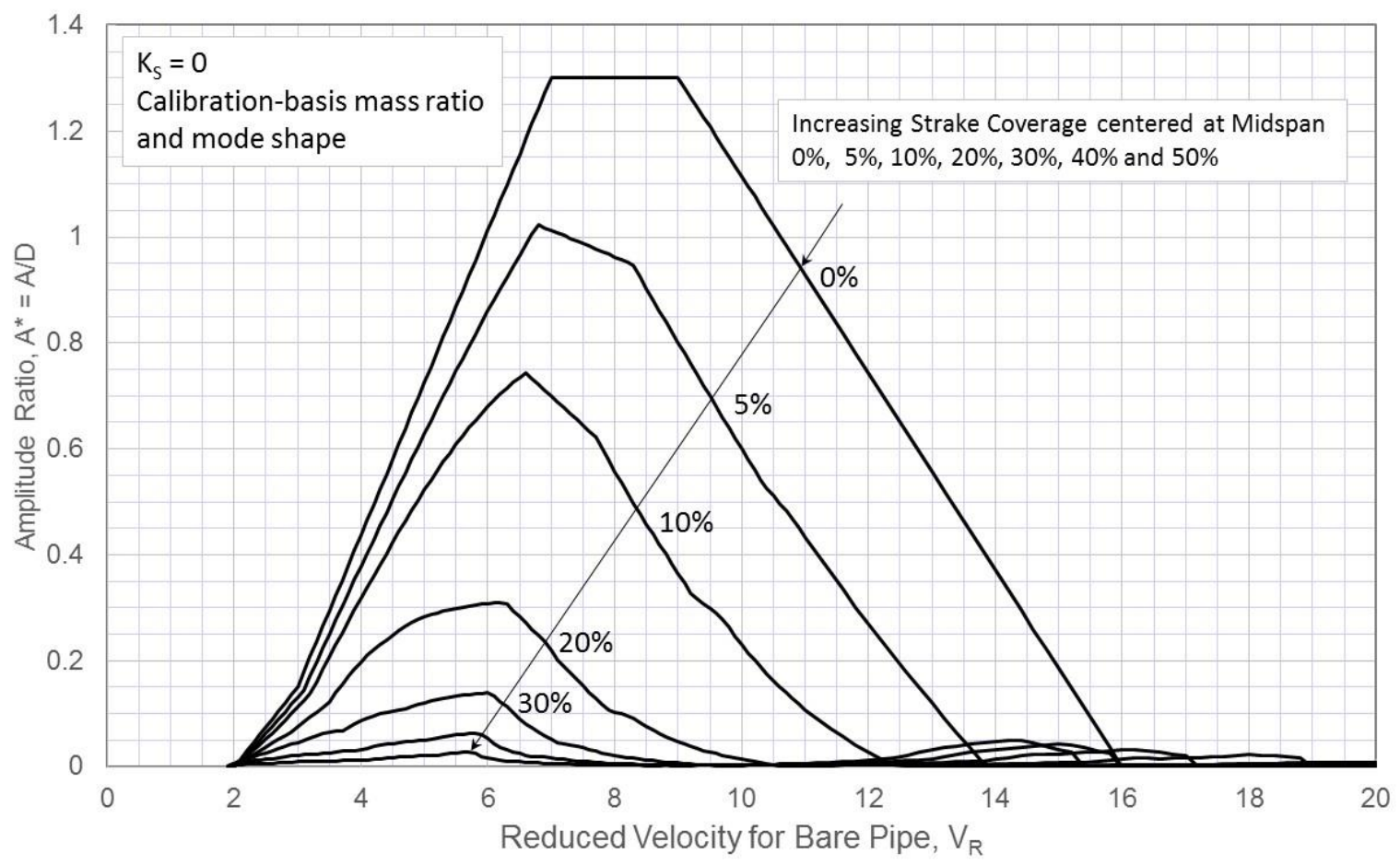

Figure 9 Effect of various levels of strake coverage on cross-flow VIV for the undamped case with the calibrationbasis mass ratio and mode shape of Table 1 .

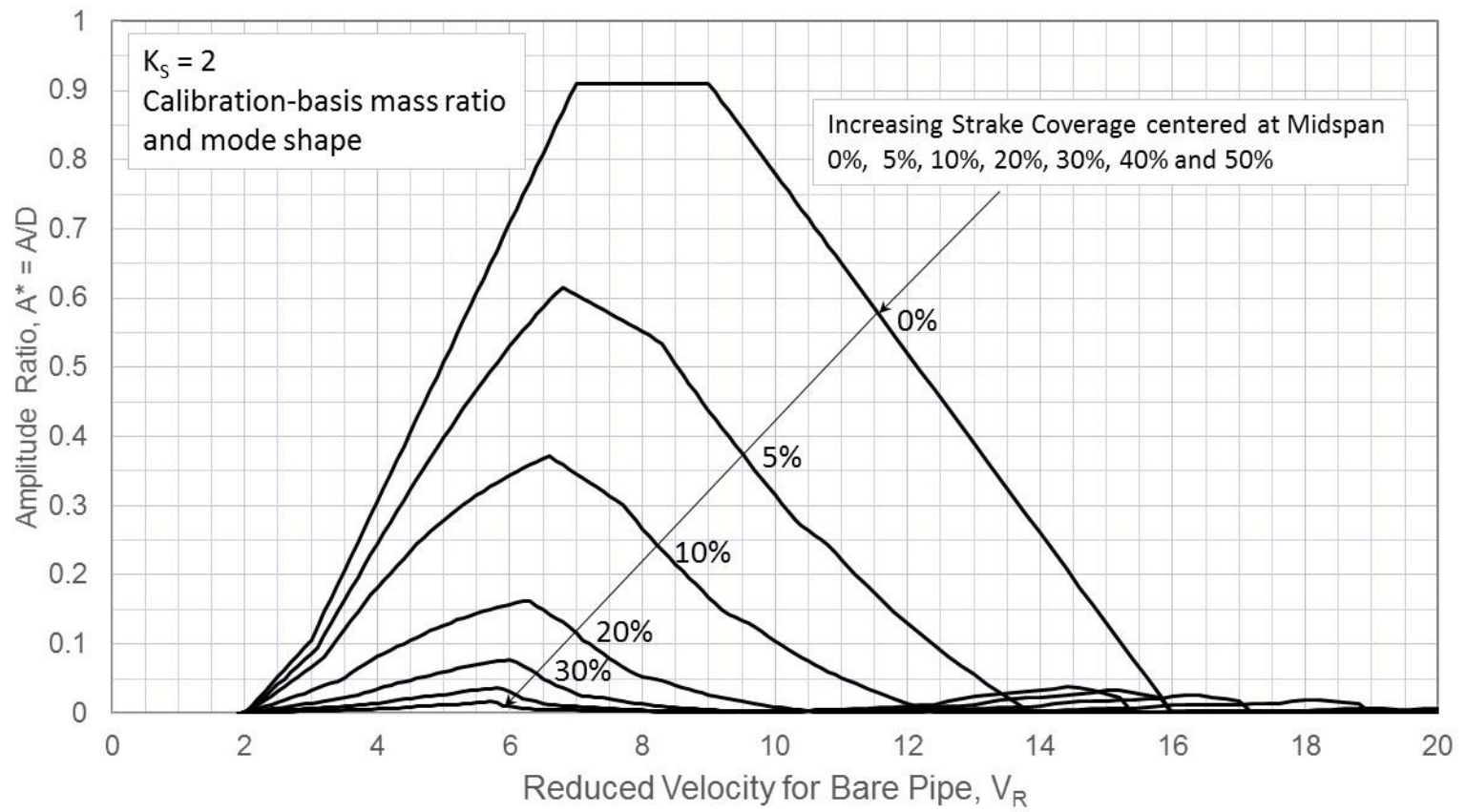

Figure $10 \quad$ As Figure 9, but for $K_{s}=2$.

\section{Closing Remarks}

This paper does not present a new theory, experimental results, nor a systematic evaluation of existing theories and experimental results. Instead it offers a practical, quantitative way to assess the effectiveness of strakes to suppress VIV in pipeline spans, building on the well-established F105 response function from [1], which is based on a broad review of experimental results that is periodically updated as new results become available. It is intended for current velocities up to the peak response of the first mode. 
The two most common ways to estimate VIV amplitudes are the response function approach typically used for pipelines and recommended in [1], and the excitation function approach such as Vivana [4], Sivana, and SHEAR7 [2], more typically used for risers. Both have advantages and disadvantages.

The advantage of the response function approach, is that it is more direct, not relying on the assumption of harmonic and uni-directional motion, and there is a larger set of experimental data from which the response function can be developed, which has been exploited in [1].

The advantage of the excitation function approach is that it can account for varying conditions along the length of the span based on theory, without requiring a set of VIV tests for every such condition. Also the mass ratio and mode shape effects are accounted for.

The inversion technique presented allows the advantages of both approaches to be exploited, by enabling the calculation of an effective excitation function from VIV tests of flexible pipes or flexibly-supported rigid cylinders at different damping ratios to be converted into an excitation function, by solving an integral equation for which an explicit numerical solution technique is given.

Sivana is merely a simplified version of Vivana [4] without propagating wave effects. It is also similar to a single-mode analysis in SHEAR7 [2], except that in Sivana the difference between flowing and still-water added mass is accounted for, whereas that difference is not addressed in [2]. Nevertheless, the Sivana energy balance condition is given in a simple, dimensionless form not found in the referenced works.

The inversion technique has been applied to the F105 cross-flow response function from [1] to get an effective bare pipe excitation function. This together with the excitation function for strakes from [19] yields a simple method to estimate the VIV response for partially strake-covered pipes that is consistent with the current practice for bare pipe, with illustrative results shown in Figure 9.

Similar results for the pure in-line case have been presented in [8] without inversion. Instead, forced vibration tests from [7] and [8], are used directly to define the excitation and added mass functions for both bare and straked pipe, respectively. In contrast to the cross-flow case, for in-line, the excitation function from the forced vibration tests already provides a good fit to flexible pipe response, as shown in [8] and in Figure 1.

The results indicate that a small amount of strake coverage is already sufficient to suppress in-line VIV, but more strakes are needed if the current velocity goes above the threshold for cross-flow.

Although inversion builds upon well-established F105 guidance in [1], care must be taken, as approximations therein that are good for one purpose may not be good for another. There remains a need for further work, including the following:

1) The F105 response function could be improved, or at least its relation to experimental data better documented in the public domain, especially at high damping values. In that process, the inversion technique presented here can help in making the best of available experimental results, e.g. to compensate for mass ratio or mode shape effects.

2) There are two issues associated with the added mass function in the form $C_{a}=C_{a}\left(f^{*}\right)$ :

a) Firstly, neglecting the effect of the vibration amplitude $\mathrm{Y}^{*}$ becomes an important drawback, e.g. at low reduced velocities when the pipe response has low amplitude and may occur at a frequency closer to the Strouhal frequency than the natural frequency of the span, implying a very high added mass that is not captured assuming added mass is a function of normalized frequency $\mathrm{f}^{*}$ only.

b) Even for flexibly supported single degree of freedom rigid cylinder tests such as [21], the response is not always harmonic, as assumed. There may be components of the response at the Strouhal frequency (implying a considerable change in added mass) as well as at the resonant frequency (for which the added mass remains close to the still-water value).

3) There remains a need for validation by tests on flexible, partially straked pipes exhibiting typical low-mode pipeline VIV response. Although there are several VIV model test campaigns where the pipe model is partially or fully straked [29-31], these exhibit high-mode mode response more typical of risers. The only such tests with low-mode response the authors are aware of are reported in [32] but they involve a different strake profile for which excitation/damping and added mass functions from forced vibration tests are not available. As a result, a direct comparison between the prediction and the model test for low mode response of a partially strake-covered span is not feasible with currently available data.

4) Finally, perfect strakes are assumed there. In practice these may be affected by marine growth, damaged, or deformed [34-36]. It remains to incorporate the effect of this on the excitation and added mass functions, so that it is accounted for in the assessment. 
A number of the above issues could be addressed by going back to the experimental data involved in the calibration of the F105 response function, and calibrating the bare pipe added mass and excitation functions directly from the data instead of indirectly from the F105 response function. The inversion technique presented in this paper facilitates that process.

\section{Acknowledgements}

This effort was partially sponsored by Shell Philippines Exploration, B.V. ("SPEX"). Permission to publish it is gratefully acknowledged. Together with [8,18,37-39] this paper arose from the original initiative of Jeffrey Rimmer at SPEX, to address the integrity threats to a major deepwater pipeline on an active seabed.

Knut Vedelt of DNVGL reviewed the manuscript and provided valuable comments. DNVGL also incorporated Sivana in a version of their "FatFree" software produced for SPEX.

However, only the authors, and not the organizations for which they work, or individuals mentioned in these acknowledgements are responsible for the content of this paper.

\section{References}

1. Det Norske Veritas (2017), "Free Spanning of Pipelines," Recommended Practice DNVGL-RP-F105, June 2017.

2. Vandiver, J.K. and Li, L., (2005), "Shear7 v 4.4 Theoretical Manual," MIT 2005.

3. Triantafyllou, M., Triantafyllou, G., David Tein, Y. S., \& Ambrose, B. D. (1999). Pragmatic riser VIV analysis. Houston, USA: Offshore Technology Conference.

4. Larsen, C.M. et al. (2008) "Vivana - Theory Manual," Marintek, Project No. 516419, Sept. 2008.

5. SINTEF Ocean (2019) "Vivana 4.16.2 Theory Manual", Tronhdeim, Norway.SINTEF Ocean (2019) "Vivana 4.16.2 Theory Manual", Tronhdeim, Norway.

6. Gopalkrishnan, R. (1993) "Vortex-Induced Forces on Oscillating Bluff Cylinders," Sc. D. Thesis, Massachusetts Institute of Technology, Department of Ocean Engineering, 1993, available at http://hdl.handle.net/1721.1/12539.

7. Aronsen, K.H., (2007), "An Experimental Investigation of In-line and Combined In-line and Cross-flow Vortex Induced Vibrations Trondheim," Thesis for the degree doctor philosophiae, Norwegian University of Science and Technology Faculty for Engineering Science and Technology, Department of Marine Technology, December 2007.

8. Wu, J., et al. (2021) "Forced Vibrations Tests for In-Line Vortex-Induced Vibration to Assess Partially Strake-Covered Pipeline Spans," Journal of Offshore Mechanics and Arctic Engineering (JOMAE), ASME, published online 10 Nov. 2020, to appear in Vol. 143, June 2021.

9. Wu, J., 2011, Hydrodynamic Force Identification from Stochastic Vortex Induced Vibration Experiments with Slender Beams, PhD Dissertation, Norwegian University of Science and Technology, Trondheim.

10. Krwawzyk, P., Bijker, R., Chen, Z., and Boersma, H., (2013), "Managing Free Spans on Dynamic Seabed," OPT 2013.

11. King, R., (1985), "Flow-Induced Vibration of Mixing Vessel Internals," Journal of Vibration, Acoustics, Stress, and Reliability in Design, ASME, Vol. 107, pp. 253-258, April 1985.

12. Taniguchi S, Miyakoshi K. Fluctuating fluid forces acting on a circular cylinder and interface with a plane wall. Experiments in Fluids, Vol 9, pp 197-204, 1990.

13. Lei C, Cheng L, Kavanagh K. Re-examination of the effect of a plane boundary on force and vortex shedding of a circular cylinder. J. Wind Eng. Ind. Aerodyn., Vol 80, pp 263- 286, 1999.

14. Tsahalis DT, Jones WT. The effect of the sea-bottom proximity on the fatigue life of suspended spans of offshore pipelines undergoing vortex-induced vibrations. Offshore Technology Conference paper 4231, 1982.

15. Tsahalis DT, Jones WT. Vortex-induced vibrations of a flexible cylinder near a plane boundary in steady flow. Offshore Technology Conference paper 3991, 1981.

16. DNVGL (2017) "Pipe-Soil Interaction for Submarine Pipelines," Det Norske Veritas, Recommended Practice, DNVGL-RP-F114, May 2017, First Issue Edition.

17. Sollund, H.A., Vedeld, K., Fyrileiv, O., (2015) "Modal Response of Free-Spanning Pipelines based on Dimensional Analysis," Applied Ocean Research 50 (2015) 13-29.

18. Peek, R., Witz, M., and Vedeld, K. (2020) "Dynamics of a Pipeline Span on an Elastic Seabed," in production, Applied Ocean Research. 
19. Spencer, D. et al. (2007) "Enabling Enhancements of Riser VIV Design Techniques through Detailed Interpretation of Test Results for VIV Suppression devices," Paper No. OTC 18973, Offshore Technology Conference, Houston Texas, 30 April - 3 May 2007.

20. Mo, K. and Solaas, F. (2002) "Ormen Lange 3D Phase II Modal Analysis", MARINTEK Report, Trondheim, Norway.

21. Blevins RD, Coughran CS. Experimental Investigation of Vortex-Induced Vibration in One and Two Dimensions With Variable Mass, Damping, and Reynolds Number. ASME. J. Fluids Eng. 2009; 131(10): 101202:10.1115/1.3222904.

22. Aglen, I. M., and Larsen, C. M. (2011) "Importance of Added Mass for the Interaction Between IL and CF Vibrations of Free Spanning Pipelines." Proceedings of the ASME 2011 30th International Conference on Ocean, Offshore and Arctic Engineering. Volume 7: CFD and VIV; Offshore Geotechnics. Rotterdam, The Netherlands. June 19-24, 2011. pp. 545-556. ASME.

23. Aglen, I. M., (2013), "VIV in Free Span Pipelines," Thesis for the degree doctor philosophiae, Norwegian University of Science and Technology Faculty for Engineering Science and Technology, Department of Marine Technology.

24. Larsen, C. M. and Lie, H.: On Hydrodynamic Coefficients for Combined Cross-Flow and In-Line Vortex Induced Vibrations. 27th International Conference of Offshore Mechanics and Arctic Engineering, OMAE 200857569, 15 p., 2008, 15-20 June, Estoril, Portugal.

25. Wu, J., Lie, H., Larsen, C. M., Liapis, S. and Baarholm, R., Vortex-induced vibration of a flexible cylinder: Interaction of the in-line and cross-flow responses. Journal of Fluids and Structures 63:238-258, May 2016.

26. Wu, J., Yin, D., Lie, H., Larsen, C. M., Baarholm, R. J., and Liapis, S. (2018). "On the Significance of the Higher-Order Stress in Riser Vortex-Induced Vibrations Responses." ASME. J. Offshore Mech. Arct. Eng. February 2019; 141(1): 011705. https://doi.org/10.1115/1.4040798.

27. Passano, E., Larsen, C. M. and Wu, J. (2010): "VIV of free spanning pipelines: Comparison of response from a semiempirical code to model tests", OMAE 2010-20330.

28. Voie, P., Wu, J., Resvanis, T.L., Larsen, Carl M., Vandiver, J. K., Triantafyllou, M, and Baarholm, R. "Consolidation of Empirics for Calculation of VIV Response." Proceedings of the ASME 2017 36th International Conference on Ocean, Offshore and Arctic Engineering. Volume 2: Prof. Carl Martin Larsen and Dr. Owen Oakley Honoring Symposia on CFD and VIV. Trondheim, Norway. June 25-30, 2017. V002T08A031. ASME. https://doi.org/10.1115/OMAE2017-61362.

29. Trim, A. D., Braaten, H., Lie, H., \& Tognarelli, M. A. (2005). Experimental investigation of vortex-induced vibration of long marine risers. Journal of Fluid and Structures(21), 335-361.

30. Lie, H., Braaten, H., Jhingran, V., Sequeiros, O., \& Vandiver, K. (2012). Comprehensive riser VIV model tests in uniform and sheared flow. Rio de Janeiro, Brazil.: the 31st International conference on Ocean, Offshore and Arctic Engineering, OMAE2012-84055.

31. Vandiver, J., Swithenbank, S., V., J., \& Jhingran, V. (2006). Gulf Stream Experiment. DeepStar 8402.

32. Huse, E.: (2001): "Ormen Lange 3D Model Tests" MARINTEK report MT51-F01.040, Trondheim, Norway.

33. Baarholm, R., and Lie, H., "Systematic parametric investigation of the efficiency of helical strakes", DOT2005, Vitoria, Brazil, November 2005.

34. Baarholm, R., and Skaugset, K. "Modelling and Characterization of Artificial Marine Growth." Proceedings of the ASME 2008 27th International Conference on Offshore Mechanics and Arctic Engineering. Volume 5: Materials Technology; CFD and VIV. Estoril, Portugal. June 15-20, 2008. pp. 863-870. ASME. https://doi.org/10.1115/OMAE2008-57587.

35. Skaugset, Kjetil, and Baarholm, Rolf. "Effect of Marine Growth on an Elastically Mounted Circular Cylinder." Proceedings of the ASME 2008 27th International Conference on Offshore Mechanics and Arctic Engineering. Volume 5: Materials Technology; CFD and VIV. Estoril, Portugal. June 15-20, 2008. pp. 855-861. ASME. https://doi.org/10.1115/OMAE2008-57586.

36. Boubenider, R., Alptunaer, K., Fourchy, P. J., \& de Wilde, J. (2008, January 1). Effectiveness of Polyethylene Helical Strakes in Suppressing VIV Responses After Sustaining High Roller Load Deformation During S-lay Installation. Offshore Technology Conference. doi:10.4043/19289-MS.

37. Ang, S.Y., Bernardo, A.C., Peek, R., Vedeld, K. and Wu, J. (2021) "Deepwater Span Management on a Mobile Seabed", planned for OMAE 2021.

38. Peek, R., Bernardo, C. A., Hong, H. M., O’Loughlin, C. D., White, D., \& Ang, S. Y. (2020, July 10). PLUCK TESTS ON OPERATING DEEPWATER PIPELINE SPANS. https://doi.org/10.31224/osf.io/qypj7

39. Peek, R. (2020) "Nonlinear Span Assessment by Amplitude-Dependent Linearization," Journal of Offshore Mechanics and Arctic Engineering (JOMAE), ASME, Vol. 42, No. 5, October 2020. 